This item was submitted to Loughborough's Research Repository by the author.

Items in Figshare are protected by copyright, with all rights reserved, unless otherwise indicated.

\title{
Kinetic equation for a soliton gas and its hydrodynamic reductions
}

PLEASE CITE THE PUBLISHED VERSION

http://dx.doi.org/10.1007/s00332-010-9080-z

PUBLISHER

(C) Springer

VERSION

AM (Accepted Manuscript)

LICENCE

CC BY-NC-ND 4.0

REPOSITORY RECORD

El, G.A., A.M. Kamchatnov, Maxim V. Pavlov, and S.A. Zykov. 2019. "Kinetic Equation for a Soliton Gas and Its Hydrodynamic Reductions". figshare. https://hdl.handle.net/2134/15335. 
This item was submitted to Loughborough's Institutional Repository (https://dspace.lboro.ac.uk/) by the author and is made available under the following Creative Commons Licence conditions.

\section{creative
commons}

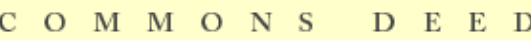

Attribution-NonCommercial-NoDerivs 2.5

You are free:

- to copy, distribute, display, and perform the work

Under the following conditions:

Attribution. You must attribute the work in the manner specified b the author or licensor.

Noncommercial. You may not use this work for commercial purposes.

No Derivative Works. You may not alter, transform, or build upon this work.

- For any reuse or distribution, you must make clear to others the license terms of this work.

- Any of these conditions can be waived if you get permission from the copyright holder.

Your fair use and other rights are in no way affected by the above.

This is a human-readable summary of the Leqal Code (the full license).

\section{Disclaimer 만}

For the full text of this licence, please go to: http://creativecommons.org/licenses/by-nc-nd/2.5/ 


\title{
Kinetic equation for a soliton gas and its hydrodynamic reductions
}

\author{
G.A. $\mathrm{El}^{1}$, A.M. Kamchatnov ${ }^{2}$, M.V. Pavlov ${ }^{3}$ and S.A. Zykov ${ }^{4}$
}

${ }^{1}$ Department of Mathematical Sciences, Loughborough University, UK

${ }^{2}$ Institute of Spectroscopy, Russian Academy of Sciences, Troitsk, Moscow Region, Russia

${ }^{3}$ Lebedev Physical Institute, Russian Academy of Sciences, Moscow

${ }^{4}$ SISSA, Trieste, Italy, and

Institute of Metal Physics, Urals Division of Russian Academy of Sciences, Ekaterinburg, Russia

\begin{abstract}
We introduce and study a new class of kinetic equations, which arise in the description of nonequilibrium macroscopic dynamics of soliton gases with elastic collisions. These equations represent nonlinear integro-differential systems and have a novel structure, which we investigate by studying in detail the class of $N$-component 'cold-gas' hydrodynamic reductions. We prove that these reductions represent integrable linearly degenerate hydrodynamic type systems for arbitrary $N$ which is a strong evidence in favour of integrability of the full kinetic equation. We derive compact explicit representations for the Riemann invariants and characteristic velocities of the hydrodynamic reductions in terms of the 'cold-gas' component densities and construct a number of exact solutions having special properties (quasi-periodic, self-similar). Hydrodynamic
\end{abstract}


symmetries are then derived and investigated. The obtained results shed the light on the structure of a continuum limit for a large class of integrable systems of hydrodynamic type and are also relevant to the description of turbulent motion in conservative compressible flows.

\section{Introduction and summary of results}

The possibility of modelling certain types of turbulent motion with the aid of the equations for weak limits of highly oscillatory dispersive compressible flows (the so-called Whitham modulation equations [42], [15], [31]) was pointed out by P.D. Lax in [30]. While this "deterministic analogue of turbulence" has obvious limitations to its possible applications to the description of hydrodynamic (incompressible) turbulent flows, it opens a whole new perspective for constructing the statistical description of purely conservative wave regimes in integrable dispersive systems by assigning appropriate probabilistic measures to the wave sequences, so that their weak limits could then be regarded as ensemble averages. Such an unconventional union of integrability and stochasticity has a natural physical motivation: nonlinear dispersive waves, while often being successfully modeled by integrable systems, could demonstrate very complex behavior calling for a statistical description characteristic of the classical turbulence theories. Recently, a closely related programme for the construction of the theory of wave turbulence in the frameworks of integrable systems has been put forward by V.E. Zakharov [49].

One of the important problems arising in this connection is the description of "soliton gases" - random distributions of solitons which can be mathematically defined in terms of generalized reflectionless potentials with shift invariant probability measure on them (see e.g. [29]). Due to isospectrality of the "primitive" microscopic evolution, the macroscopic dynamics of a homogeneous soliton gas is trivial (for the so-called 'strongly integrable' systems, such as the Korteweg - de Vries (KdV), nonlinear Schrödinger (NLS) or Kadomtsev-Petviashvili (KP-II) equations - see [49]), namely, all the statistical characteristics can be specified arbitrarily at the initial moment and remain unchanged in time. However, if the soliton gas 
is spatially inhomogeneous, i.e. if the probability distribution function depends on the space coordinate, then nontrivial macroscopic dynamics can occur due to phase shifts of individual solitons in their collisions with each other. An approximate kinetic equation describing spatial evolution of the soliton distribution function in a rarefied gas of the KdV solitons, when these phase shifts can be taken into account explicitly, was derived by Zakharov back in 1971 [46].

Generalization of Zakharov's kinetic equation to the case of a soliton gas of finite density has been made possible rather recently [6] and required consideration of the continuum thermodynamic limit of the Whitham modulation equations associated with finite-gap potentials. In the thermodynamic limit, the nonlinear interacting wave modes transform into randomly distributed localised states (solitons) and the modulation system assumes the form of a nonlinear kinetic equation. This new kinetic equation was extended, using physical reasoning, in [7] to other integrable systems with two-particle elastic interactions of solitons (i.e. when multi-particle effects are absent).

The kinetic equation for solitons in general form represents a nonlinear integro-differential system

$$
\begin{aligned}
& f_{t}+(s f)_{x}=0, \\
& s(\eta)=S(\eta)+\frac{1}{\eta} \int_{0}^{\infty} G(\eta, \mu) f(\mu)[s(\mu)-s(\eta)] d \mu .
\end{aligned}
$$

Here $f(\eta) \equiv f(\eta, x, t)$ is the distribution function and $s(\eta) \equiv s(\eta, x, t)$ is the associated transport velocity. The (given) functions $S(\eta)$ and $G(\eta, \mu)$ do not depend on $x$ and $t$. The function $G(\eta, \mu)$ is assumed to be symmetric, i.e. $G(\eta, \mu)=G(\mu, \eta)$. The choice

$$
S(\eta)=4 \eta^{2}, \quad G(\eta, \mu)=\log \left|\frac{\eta-\mu}{\eta+\mu}\right|
$$

corresponds to the $\mathrm{KdV}$ soliton gas [6], where the $\mathrm{KdV}$ equation is taken in the canonical form

$$
\phi_{t}-6 \phi \phi_{x}+\phi_{x x x}=0
$$

In the $\mathrm{KdV}$ context, $\eta \geq 0$ is a real-valued spectral parameter (to be precise, before the passage to the continuum limit one has $\lambda_{k}=-\eta_{k}^{2}$, where $\lambda_{k}, k=1, \ldots, N$ are the dis- 
crete eigenvalues of the Schrödinger operator), thus the function $f(\eta, x, t)$ is the distribution function of solitons over spectrum so that $\kappa=\int_{0}^{\infty} f(\eta) d \eta=\mathcal{O}(1)$ is the spatial density of solitons. If $\kappa \ll 1$, the first order approximation of (1), (2) yields Zakharov's kinetic equation for a dilute gas of KdV solitons [46] (see equations (28), (29) below).

The quantity $S(\eta)$ in (1) has a natural meaning of the velocity of an isolated (free) soliton with the spectral parameter $\eta$ and the function $\frac{1}{\eta} G(\eta, \mu)$ is the expression for a phase shift of this soliton occurring after its collision with another soliton having the spectral parameter $\mu<\eta$. Then $s(\eta, x, t)$ acquires the meaning of the self-consistently defined mean local velocity of solitons with the spectral parameter close to $\eta$ (see [7]).

Theory of nonlocal kinetic equations of the form (1) is not developed yet. Possible approaches to their treatment were discussed in [2] in connection with special classes of exact solutions for the Boltzmann kinetic equation for Maxwellian particles. The derivation of (1), (2) as a certain (albeit singular) limit of the integrable KdV-Whitham system suggests that this new kinetic equation is also an integrable system, at least for special choices of functions $G(\eta, \mu)$. A natural question arising in this connection is: what is the exact meaning of integrability for the equations of the type (1)?

Integrability of kinetic equations has been the object of intensive studies in recent decades. For instance, integrability of the collisionless Boltzmann equation (which is sometimes called the Vlasov equation) can be defined in terms of two other closely connected (even equivalent in some sense) objects: the Benney hydrodynamic chain [3], [47], [17] and the dispersionless limit of the Kadomtsev-Petviashvili equation $([27,28]$. It turns out that all these three different nonlinear partial differential equations possess the same infinite set of $N$-component hydrodynamic reductions parameterized by $N$ arbitrary functions of a single variable $[18,19]$ (we note that the solutions to these $N$-component reductions are parameterized, in their turn, by another $N$ arbitrary functions of a single variable). This property was used in [12, 13] (see also [48], [14], [20], [34]) when introducing the integrability criterion for a wide class of kinetic equations, corresponding hydrodynamic chains and $2+1$ quasilinear equations. Moreover, it was proved in [36] that the existence of at least one $N$-component hydrodynamic reduction written in the so-called symmetric form is sufficient for integrability in the sense 
of [12]. Another possible approach to analyse an integrable kinetic equation is to use the fact that it possesses infinitely many particular solutions determined by the corresponding hydrodynamic reductions (see [34] for details).

The distinctive feature of the kinetic equation (1) is its nonlocal structure, which represents an obstacle to the direct application to it of the approaches developed in [36] and [34]. For instance, the possibility of an explicit construction of symmetric hydrodynamic reductions, and even the existence of such reductions for (1), are open questions at the moment. In this paper, we study a particular, yet probably the most important from the viewpoint of capturing the essential properties of the full equation, family of the 'cold-gas' $N$-component hydrodynamic reductions of (1) obtained via the delta-function ansatz for the distribution function $f(\eta, x, t)=\sum_{i=1}^{N} f^{i}(x, t) \delta\left(\eta-\eta_{i}\right)$, where $\eta_{N}>\eta_{N-1}>\cdots>\eta_{1}>0$ are arbitrary numbers. Then the velocity distribution $s(\eta, x, t)$ over the 'spectrum' becomes a discrete set of functions $\left\{s^{i}(x, t): s^{i}=s\left(\eta_{i}, x, t\right), i=1, \ldots, N\right\}$ and the sought reductions family assumes the form of a system of hydrodynamic conservation laws

$$
u_{t}^{i}=\left(u^{i} v^{i}\right)_{x}, \quad i=1, \ldots, N
$$

where the the 'densities' $u^{i}=\eta_{i} f^{i}(x, t)$ and the velocities $v^{i}=-s^{i}(x, t)$ are related algebraically:

$$
v^{i}=\xi_{i}+\sum_{m \neq i} \epsilon_{i m} u^{m}\left(v^{m}-v^{i}\right), \quad \epsilon_{i k}=\epsilon_{k i}
$$

Here

$$
\xi_{i}=-S\left(\eta_{i}\right), \quad \epsilon_{i k}=\frac{1}{\eta_{i} \eta_{k}} G\left(\eta_{i}, \eta_{k}\right), \quad i \neq k
$$

Despite the deceptively simple form of system (4), (5), an attempt of the analysis of its integrability properties by employing standard methods of the theory of hydrodynamic type systems (verification of the Haantjes tensor vanishing, computation of the Riemann invariants in terms of the densities of conservation laws, establishing the semi-Hamiltonian property etc. - see, e.g. [37]) reveals serious technical problems already for a modest $N=4$. The reason for such unexpected difficulties in the apparently straightforward procedure lies in the fact that the existing theory heavily relies on the knowledge of the explicit dependence of the coefficient matrix of the hydrodynamic type system on field variables 
while the dependence $v^{i}(\mathbf{u})$ in (4) is given implicitly by algebraic system (5). It turns out that the resolution of this system for $v^{i}$ using standard computer algebra packages becomes notoriously resource consuming with the growth of $N$ and does not hold any promise of getting structurally transparent results for the Riemann invariants and characteristic velocities. This makes the standard direct route completely prospectless from the viewpoint of proving integrability of (4), (5) and obtaining explicit analytic results for an arbitrary $N$. To deal with the specific structure of system (4), (5) we develop in this paper a new approach, which has enabled us to perform the complete analysis of its integrability for an arbitrary $N$ and, in particular, to derive compact and elegant representations for the Riemann invariants and characteristic velocities.

The main results of the paper can be summarized as follows:

- We prove that reductions (4), (5) represent linearly degenerate integrable systems of hydrodynamic type for arbitrary $N$. This is done by proving the existence of a certain representation of the densities $u^{i}$ and velocities $v^{i}$ in terms of the so-called Stäckel matrix which depends on $N$ functions $r^{i}(x, t)$, which are the Riemann invariants of equations (4), (5). We also prove that system (4), (5) belongs to the Egorov class (see Def. 7.1 in Section 7). Moreover, as a by-product of our analysis, we conclude that the system under study is the only (up to unessential transformations) system of hydrodynamic type which is simultaneously Egorov and linearly degenerate. The characteristic velocities, conservation law densities and symmetries (commuting flows) for such systems are fixed by $N(N-1) / 2$ symmetric constants $\epsilon_{i k}, i \neq k$, and $N$ constants $\xi_{i}$ (i.e. by $N(N+1) / 2$ constants in total).

- We derive an explicit Riemann invariant representation of system (4), (5),

$$
r_{t}^{i}=v^{i}(\mathbf{r}) r_{x}^{i}, \quad i=1, \ldots, N
$$

where the Riemann invariants $r^{i}$ are expressed in terms of the component densities $u^{1}, u^{2}, \ldots, u^{N}$ as

$$
r^{i}=-\frac{1}{u^{i}}\left(1+\sum_{m \neq i} \epsilon_{i m} u^{m}\right), \quad i=1, \ldots, N
$$


and for the characteristic velocities $v^{i}(\mathbf{r})$ we obtain

$$
v^{i}=\frac{1}{u^{i}} \sum_{m=1}^{N} \xi_{m} \beta_{i m}, \quad \text { where } \quad u^{i}=\sum_{m=1}^{N} \beta_{i m} .
$$

Here the matrix $\boldsymbol{\beta}=-\boldsymbol{\epsilon}^{-1}$ where the off-diagonal elements of the symmetric matrix $\boldsymbol{\epsilon}$ are fixed by system (5) while the diagonal elements are defined as $\epsilon_{i i}=r^{i}$. Remarkably, the off-diagonal symmetric elements of the matrix $\boldsymbol{\beta}$ are nothing than the rotation coefficients of the curvilinear conjugate coordinate net associated with system (6). We also note that the second formula in (8) is in fact the inversion of the explicit representation (7). Importantly, the characteristic velocities in (6) coincide with the transport velocities in the conservation laws (4) — this is the consequence of linear degeneracy of system (4), (5).

- We construct the full set of commuting flows to (4), (5), of which $N-2$ are linearly degenerate. This has allowed us, in particular, to obtain the family of quasi-periodic solutions

$$
x+\xi_{i} t=\int^{r^{i}} \frac{\xi d \xi}{\sqrt{R_{K}(\xi)}}+\sum_{m \neq i} \epsilon_{i m} \int^{r^{m}} \frac{d \xi}{\sqrt{R_{K}(\xi)}}, \quad i=1,2, \ldots, N
$$

where

$$
R_{K}(\xi)=\prod_{n=1}^{K}\left(\xi-E_{n}\right)
$$

and $E_{1}<E_{2}<\cdots<E_{K}$ are real constants $(K=2 N+1$ if $N$ is odd and $K=2 N+2$ if $N$ is even)

- We show that for the special case $N=3$ there exists a family of similarity solutions to (6), (8) having the form $\tilde{r}^{i}=t^{-\alpha} l^{i}(x / t), i=1,2,3, \alpha \neq 0$, where each $\tilde{r}^{i}$ is a certain rational function of the corresponding Riemann invariant $r^{i}(7)$ (and hence, is also a Riemann invariant). These solutions are found in an implicit (hodograph) form. For $N>3$ such solutions generally do not exist.

Integrability, for arbitrary $N$, of the class of the hydrodynamic reductions studied in this paper is a strong evidence in favour of integrability of the full nonlocal kinetic equation (1), at least for certain choices of the functions $S(\lambda)$ and $G(\lambda, \mu)$ in the integral closure equation. 
Of course, such an outcome does not look surprising for the particular choice (2) of $S(\lambda)$ and $G(\lambda, \mu)$ corresponding to the thermodynamic limit of the integrable KdV-Whitham equations but our analysis suggests that the general integro-differential kinetic equation (1) is a representative of a whole new unexplored class of integrable equations with potentially important physical applications.

The structure of the paper is as follows. In Section 2 we outline the derivation of the kinetic equations for the gas of the KdV solitons following the thermodynamic limit procedure of [6] and extending it to the entire KdV-Whitham hierarchy. We then introduce the generalized equation (1), and in Section 3 consider its $N$-component 'cold-gas' hydrodynamic reductions (4), (5) having the form of hydrodynamic conservation laws. We then formulate our main Theorem 3.1 stating that the hydrodynamic reductions under study are linearly degenerate and integrable (in Tsarev's generalised hodograph sense) hydrodynamic type systems for any $N$. Section 4 is devoted to the account of the main results of the theory of linearly degenerate hydrodynamic type systems. In Section 5 we prove the statement of the main Theorem 3.1 for the case $N=3$ by explicitly constructing the corresponding Stäckel matrix and presenting expressions for the Riemann invariants and characteristic velocities in terms of the conserved component densities. We also construct two distinguished families of exact solutions (self-similar and quasi-periodic) to the 3-component reduction. In Section 6, the existence of the Riemann invariant parametrization of the cold-gas hydrodynamic reduction, via a single Stäckel matrix, is proved for arbitrary $N$, which enables us to complete the proof of the main Theorem 3.1 for a general case. In Section 7, we derive explicit expressions (7) and (8) for the Riemann invariants and characteristic velocities in terms of the component densities. And at last, in Section 8 we derive hydrodynamic symmetries (commuting flows) of the $N$-component hydrodynamic reductions under study and then extract the family of linearly degenerate commuting flows. We conclude in Section 9 with a general outlook and perspectives arising from our study. 


\section{Kinetic equation for a soliton gas as the thermody- namic limit of the Whitham modulation system}

We start with an outline of the derivation of the kinetic equation for the gas of the $\mathrm{KdV}$ solitons as the thermodynamic limit of the KdV-Whitham system following [6]. We then naturally extend this derivation to the entire Whitham-KdV hierarchy.

Let us consider the Whitham modulation system associated with the $N$-gap potentials $\phi_{N}(x, t)$ of the $\mathrm{KdV}$ equation (3). This system is most conveniently represented as a single generating equation in the form [15]:

$$
\left(d p_{N}\right)_{t}=\left(d q_{N}\right)_{x}
$$

where $d p_{N}$ and $d q_{N}$ are the quasimomentum and quasienergy differentials defined on the two-sheeted hyperelliptic Riemann surface of genus $N$ :

$$
\begin{gathered}
\Gamma: \quad \mu^{2}(\lambda)=\prod_{j=1}^{2 N+1}\left(\lambda-\lambda_{j}\right), \quad \lambda \in \mathbb{C}, \quad \lambda_{j} \in \mathbb{R} . \\
\lambda_{1}<\lambda_{2}<\cdots<\lambda_{2 N}<\lambda_{2 N+1},
\end{gathered}
$$

with cuts along spectral bands $\left[\lambda_{1}, \lambda_{2}\right], \ldots\left[\lambda_{2 j-1}, \lambda_{2 j}\right], \ldots,\left[\lambda_{2 N+1}, \infty\right]$. We introduce the canonical system of cycles on $\Gamma$ as follows (see Fig. 1): the $\alpha_{j}$-cycle surrounds the $j$-th cut clockwise on the upper sheet, and the $\beta_{j}$ - cycle is canonically conjugated to $\alpha_{j}$ 's such that the closed contour $\beta_{j}$ starts at $\lambda_{2 j}$, goes to $+\infty$ on the upper sheet and returns to $\lambda_{2 j}$ on the lower sheet.

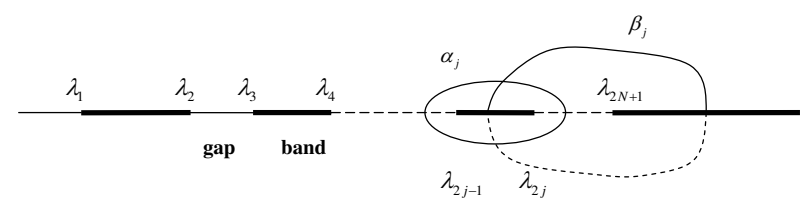

Figure 1: The canonical system of cycles on the hyperelliptic Riemann surface of genus $N$.

The meromorphic differentials $d p_{N}$ and $d q_{N}$ are uniquely defined by their asymptotic behavior near $\lambda=-\infty$ :

$$
-\lambda \gg 1: \quad d p_{N} \sim-\frac{d \lambda}{(-\lambda)^{1 / 2}}, \quad d q_{N} \sim(-\lambda)^{1 / 2} d \lambda
$$


and the normalization

$$
\oint_{\beta_{i}} d p_{N}=0, \quad \oint_{\beta_{i}} d q_{N}=0, \quad i=1, \ldots, N ; \quad c_{N}=-\frac{1}{2} \sum_{j=1}^{2 N+1} \lambda_{j} .
$$

The integrals of $d p_{N}$ and $d q_{N}$ over the $\alpha$ - cycles give the components of the wave number and the frequency vectors respectively

$$
\oint_{\alpha_{j}} d p_{N}(\lambda)=k_{j}\left(\lambda_{1}, \ldots, \lambda_{2 N+1}\right), \quad \oint_{\alpha_{j}} d q_{N}(\lambda)=\omega_{j}\left(\lambda_{1}, \ldots, \lambda_{2 N+1}\right), \quad j=1, \ldots, N .
$$

Let $\lambda_{1}=-1, \lambda_{2 N+1}=0$. Following Venakides [44] we introduce a lattice of points

$$
1 \approx \eta_{1}>\eta_{2}>\ldots>\eta_{N} \approx 0
$$

where

$$
-\eta_{j}^{2}=\frac{1}{2}\left(\lambda_{2 j-1}+\lambda_{2 j}\right)
$$

are the centres of bands.

We now assume that the spectral bands are distributed such that one can introduce two positive continuous functions on $[0,1]$ :

1. The normalized density of bands $\varphi(\eta)$ :

$$
\varphi(\eta) d \eta \approx \frac{\text { number of lattice points in }(\eta, \eta+d \eta)}{N} .
$$

That is,

$$
\varphi\left(\eta_{j}\right)=\frac{1}{N\left(\eta_{j}-\eta_{j+1}\right)}+O\left(\frac{1}{N}\right), \quad \int_{0}^{1} \varphi(\eta) d \eta=1, \quad \eta^{2}=-\lambda \in[0,1] .
$$

2. The normalized logarithmic band width $\gamma(\eta)$ :

$$
\gamma\left(\eta_{j}\right)=-\frac{1}{N} \log \delta_{j}+O\left(\frac{1}{N}\right), \quad \delta_{j}=\lambda_{2 j}-\lambda_{2 j-1}
$$

The functions $\varphi(\eta)$ and $\gamma(\eta)$ asymptotically define the local structure of the Riemann surface $\Gamma$ (11) for $N \gg 1$. In other words, instead of $2 N+1$ discrete parameters $\lambda_{j}$ we have two continuous functions of $\eta$ on $[0,1]$ which do not depend on $x, t$ on the scale of the typical change of $\lambda_{j}$ 's in (10), say $\Delta x \sim \Delta t \sim l$. 
The existence of the continuous distributions $\varphi(\eta)$ and $\gamma(\eta)$ implies the following bandgap scaling for $N \gg 1$ :

$$
\left|\operatorname{gap}_{j}\right| \sim \frac{1}{\varphi\left(\eta_{j}\right) N}, \quad\left|\operatorname{band}_{j}\right| \sim \exp \left\{-\gamma\left(\eta_{j}\right) N\right\}, \quad j=1, \ldots, N
$$

Introduction of the distribution (19) is motivated by the structure of the spectrum of Hill's operator in the semi-classical limit [41], [43] although the scaling (19) alone, of course, does not imply exact periodicity of the (finite-gap) potential.

The scaling (19) has an important property: it preserves the finiteness of the integrated density of states as $N \rightarrow \infty$. The integrated density of states is defined in terms of the real part of the quasimomentum integral (see [24]):

$$
\mathcal{N}_{N}(\lambda)=\frac{1}{\pi} \operatorname{Re} \int_{-1}^{\lambda} d p_{N}\left(\lambda^{\prime}\right), \quad \lambda \in[-1,0]
$$

Now, using (14) one can readily see that

$$
\mathcal{N}_{N}(\lambda)=\frac{1}{2 \pi} \sum_{j=1}^{M} k_{j} \quad \text { if } \quad \lambda \in\left[\lambda_{2 M}, \lambda_{2 M+1}\right], \quad M=1, \ldots, N,
$$

which is a particular (finite-gap) case of the general gap-labeling theorem for quasi-periodic potentials [24]. It is not difficult to show that the scaling (19) implies that $k_{j} \sim 1 / N$ so the total density of states

$$
\mathcal{N}_{N}(0)=\frac{1}{2 \pi} \sum_{j=1}^{N} k_{j}
$$

remains finite in the limit as $N \rightarrow \infty$. For this reason we shall call the continuum limit as $N \rightarrow \infty$, defined on the spectral scaling (19), the thermodynamic limit.

We shall not be concerned here with the existence and the exact meaning of the thermodynamic limit for the finite-gap potentials $u_{N}(x, t)$ (which is a separate interesting problem closely connected with Venakides' continuum limit of theta-functions [44]) but shall rather directly consider this limit for the associated Whitham system (10). It is however, instructive to note that it follows from (19) that in the thermodynamic limit the band/gap ratio vanishes for each oscillating mode (i.e. $k_{j} \rightarrow 0 \forall j=1,2 \ldots, N$ ), so the thermodynamic limit of the sequence of finite-gap potentials associated with the spectral scaling (19) is essentially an infinite-soliton limit. It was proposed in [8] that this limiting potential should be 
described in terms of ergodic random processes and can be viewed as a homogeneous soliton gas (or homogeneous soliton turbulence - depending on which of the two "identities" of a soliton is emphasized: the particle or the wave one). Then it is natural to suppose that the same thermodynamic limit for the associated Whitham system should describe macroscopic evolution of the spatially inhomogeneous soliton gas. Indeed, as we shall see, the thermodynamic limit of the Whitham equations turns out to be consistent (in the small-density limit) with the kinetic equation for solitons derived by Zakharov [46] using the inverse scattering problem formalism.

We first note that $\mathcal{N}_{N}(\lambda)$ defined by (20) is a monotone increasing positive function so $d \mathcal{N}_{N}(\lambda)$ is a measure supported on the spectrum of the finite-gap potential $u_{N}(x)$ [24]. Next we introduce a 'temporal' analogue of the density of states (20) by the formula

$$
\mathcal{V}_{N}(\lambda)=\frac{1}{\pi} R e \int_{-1}^{\lambda} d q_{N}\left(\lambda^{\prime}\right), \quad \lambda \in[-1,0]
$$

Then integration of the generating modulation equation (10) on the real axis of $\lambda$ from -1 to $-\eta^{2} \in[-1,0]$ yields

$$
\partial_{t} d \mathcal{N}_{N}\left(-\eta^{2}\right)=\partial_{x} d \mathcal{V}_{N}\left(-\eta^{2}\right), \quad \eta \in[0,1]
$$

Thus the finite-gap Whitham-KdV system can be regarded as the system governing the evolution of the spectral measure.

Now we consider the thermodynamic limits of $d \mathcal{N}_{N}$ and $d \mathcal{V}_{N}$ which we denote as

$$
d \mathcal{N}_{N} \rightarrow \pi f(\eta) d \eta, \quad d \mathcal{V}_{N} \rightarrow-\pi f(\eta) s(\eta) d \eta \quad \text { as } \quad N \rightarrow \infty
$$

where the limit is taken on the thermodynamic spectral scaling (19). Since $\pi f(\eta) d \eta$ is the limiting spectral measure, the function $f(\eta)$ has the natural meaning of the distribution function of the solitons over the spectrum (the meaning of the function $s(\eta)$ will become clear soon). The functions $f(\eta)$ and $s(\eta)$ were shown in [8], [6] to be expressed in terms of the ratio $\sigma(\eta)=\varphi(\eta) / \gamma(\eta)$ of the lattice distribution functions (17), (18) by certain integral equations, which are then combined into a single equation directly connecting $f(\eta)$ and $s(\eta)$ 
[6]:

$$
s(\eta)=4 \eta^{2}+\frac{1}{\eta} \int_{0}^{1} \log \left|\frac{\eta+\mu}{\eta-\mu}\right| f(\mu)[s(\eta)-s(\mu)] d \mu .
$$

We stress that in the continuum (thermodynamic) limit given by equations (25), (26) the explicit dependence of the density of states on the discrete spectral branch points $\lambda_{j}$ disappears. The only 'reminder' of the hyperelliptic Riemann surface $\Gamma$ (11) is the kernel $\ln |\eta+\mu| /|\eta-\mu|$ which arises as the continuum limit of the off-diagonal elements of the period matrix $\mathbf{B}$ of the Riemann theta-function $\Theta_{N}(x, t \mid \mathbf{B})$ defining, via the Its-Matveev formula, the finite-gap potential (see [44] and [6]).

Thus, integral equation (26) can be viewed as a local (in the $x, t$-plane) relationship between the functions $f(\eta)$ and $s(\eta)$ characterizing the soliton gas. Let $l \gg 1$ be the characteristic length at which the change of functions $f(\eta), s(\eta)$ is small (of order $1 / l \ll 1$ ). Next, in the spirit of the modulation theory (see [42], [15]) we assume that on a larger spatiotemporal scale, $\Delta x \gg l, \Delta t \gg l$, we have $f(\eta) \equiv f(\eta, x, t), s(\eta) \equiv s(\eta, x, t)$ and postulate, using (25), that

$$
\partial_{t} d \mathcal{N}_{N} \rightarrow \pi \partial_{t} f(\eta, x, t) d \eta, \quad \partial_{x} d \mathcal{V}_{N} \rightarrow-\pi \partial_{x}[f(\eta, x, t) s(\eta, x, t)] d \eta .
$$

Then modulation equation (24) assumes the form of a conservation equation for $f$,

$$
f_{t}+(s f)_{x}=0,
$$

which is clearly the expression of the isospectrality of the KdV evolution. Since $\rho(x, t)=$ $\int_{0}^{1} f d \eta$ is the density of solitons the quantity $s(\eta, x, t)$ can naturally be interpreted as the velocity of the soliton gas (or, more precisely, the velocity of a 'trial' soliton with the spectral parameter $\lambda=-\eta^{2}$ - see [23]). One can see from (26) that this velocity differs from the velocity $4 \eta^{2}$ of the free soliton with the same spectral parameter. This difference is obviously due to the collisions of the 'trial' $\eta$-soliton with other ' $\mu$ ' - solitons in the soliton gas. Indeed, for small densities $\rho=\int f d \eta \ll 1$ one can consider the second term in (26) as a small correction to the free-soliton velocity and obtain that to the first order in $\rho$

$$
s(\eta) \approx 4 \eta^{2}+\frac{1}{\eta} \int_{0}^{1} \ln \left|\frac{\eta+\mu}{\eta-\mu}\right| f(\mu)\left[4 \eta^{2}-4 \mu^{2}\right] d \mu,
$$


which is Zakharov's expression for the average velocity of a trial soliton in a rarefied soliton gas, obtained in [46] by taking into account the change in the soliton position due to phase shifts in its pairwise collisions with other solitons. We would like to emphasize crucial difference between the mathematical structure of Zakharov's asymptotic formula (29), which is an explicit expression for the trial soliton velocity $s(\eta)$ in terms of the spectral distribution function $f(\eta)$, and formula (26) which is a non-perturbative integral equation for $s(\eta)$ for a given $f(\eta)$.

Equations (28) and (26) thus provide a self-consistent kinetic description of the KdV soliton gas of finite density. We note that the upper limit in the integrals in (26), (29) can be replaced by $+\infty$ to make the kinetic equation independent on the original spectral lattice normalization (15).

The outlined procedure of the thermodynamic limit can be readily extended to the entire Whitham-KdV hierarchy,

$$
\left(d p_{N}\right)_{t_{n}}=\left(d q_{N}^{(n)}\right)_{x}, \quad n \in \mathbb{N}
$$

where $n$ is the number of the "higher" Whitham-KdV equation in the hierarchy (the original modulation equation (10) corresponding to the $\mathrm{KdV}$ equation itself has the number $n=1$ ) and $t_{n}$ is the corresponding "higher" time, so that $\left(d p_{N}\right)_{t_{n} t_{m}}=\left(d p_{N}\right)_{t_{m} t_{n}}$ for all $n \neq m$. The meromorphic differential $d q_{N}^{(n)}$ is uniquely defined by its asymptotic behavior near $\lambda=-\infty$,

$$
d q^{(n)} \sim(-\lambda)^{n-1 / 2} d \lambda
$$

and the normalization

$$
\oint_{\beta_{j}} d q^{(n)}(\lambda)=0, \quad j=1, \ldots, N
$$

analogous to (13).

Now, applying the outlined above procedure of the thermodynamic limiting transition to equation (30) we obtain the same transport equation (28) for the distribution function $f(\eta, x, t)$

$$
f_{t_{n}}+\left(s_{n} f\right)_{x}=0
$$


while the integral closure equation for $s_{n}$ assumes the form

$$
s_{n}(\eta)=C_{n} \eta^{2 n}+\frac{1}{\eta} \int_{0}^{1} \log \left|\frac{\eta+\mu}{\eta-\mu}\right| f(\mu)\left[s_{n}(\eta)-s_{n}(\mu)\right] d \mu,
$$

where $C_{n}$ are certain constants whose specific values won't be required below. Moreover, since the characteristic speeds of the commuting KdV-Whitham flows, and, therefore, the corresponding transport velocities $s_{n}$ in the thermodynamic limit equation (34), are defined up to a constant factor, hereafter one can assume $C_{n}$ to be arbitrary constants.

We note that equation (34) differs from (26) only in the first term corresponding to the free-soliton velocity. Also note that the 'phase-shift' logarithmic kernel in the integral equation (34) is the same for all $n$, which is not surprising as the whole finite-gap WhithamKdV hierarchy (30) is associated with the same Riemann surface, i.e. with the same period matrix $\mathbf{B}$ responsible for the form of the integral kernel in the limit.

Now it is only natural to consider a generalization of the derived kinetic equations (33), (34) by introducing in (34) an arbitrary function $S(\eta)$ instead of the free-soliton velocity term and an arbitrary symmetric function $G(\eta, \mu)$ instead of the logarithmic "phase-shift kernel' in the integral term. Also, for generality we replace the upper limit of integration in the closure equation $(26)$ by $+\infty$. As a result, we arrive at the generalized kinetic equation (1), which will be our main concern in the remainder of the paper.

\section{3 'Cold-gas' hydrodynamic reductions}

We introduce an $N$-component 'cold-gas' ansatz for the distribution function $f(\eta, x, t)$ :

$$
f=\sum_{i=1}^{N} f^{i}(x, t) \delta\left(\eta-\eta_{i}\right)
$$

where $\eta_{N}>\eta_{N-1}>\cdots>\eta_{1}>0$ are arbitrary numbers and $f^{i}(x, t), n=1, \ldots, N$ are unknown functions.

Before we proceed with the analysis of mathematical consequences of this 'cold-gas' ansatz it is instructive to say a couple of words about its physical meaning (see [7]). To be definite, we shall refer to the KdV case here. The distribution (35) represents an idealized description 
of the distribution function in a soliton gas with the solitons having their spectral parameters $\eta$ distributed in narrow vicinities of $N$ discrete values $\eta_{i}$. As a matter of fact, owing to nondegeneracy of discrete spectrum of the linear Schrödinger operator, all individual spectral parameters within the $i$-th component must be different. The soliton positions in such a 'quasi-monochromatic' component of the soliton gas are statistically independent which results in the Poisson distribution with the mean density $f_{i}$ for the number of solitons in a unit space interval (the Poisson distribution naturally arises in the thermodynamic limit of finite-gap potentials [8]). It is also clear that one can neglect the effect of the interactions between the solitons belonging to the same gas component compared with the cross-component interactions (the typical time of the interactions between solitons with close values of the spectral parameter is much larger than when these parameters are mutually spaced within the spectral interval — see, e.g., [33]). This will be shown below to have important mathematical consequences.

Substitution (35) reduces (1) to a system of hydrodynamic conservation laws,

$$
u_{t}^{i}=\left(u^{i} v^{i}\right)_{x}, \quad i=1, \ldots, N
$$

where the component 'densities' $u^{i}$ and the velocities $v^{i}$ defined as

$$
u^{i}(x, t)=\eta_{i} f^{i}(x, t), \quad v^{i}(x, t)=-s\left(\eta_{i}, x, t\right),
$$

are related algebraically

$$
v^{i}=\xi_{i}+\sum_{m \neq i} \epsilon_{i m} u^{m}\left(v^{m}-v^{i}\right), \quad \epsilon_{i k}=\epsilon_{k i} .
$$

Here

$$
\xi_{i}=-S\left(\eta_{i}\right), \quad \epsilon_{i k}=\frac{1}{\eta_{i} \eta_{k}} G\left(\eta_{i}, \eta_{k}\right) \quad i \neq k .
$$

Note that the elements $\epsilon_{i i}$ are not defined.

In a two-component case, the above algebraic system (38) can be easily resolved for $u^{1,2}$ in terms of $v^{1,2}$ :

$$
u^{1}=\frac{1}{\epsilon_{12}} \frac{v^{2}-\xi_{2}}{v^{1}-v^{2}}, \quad u^{2}=\frac{1}{\epsilon_{12}} \frac{v^{1}-\xi_{1}}{v^{2}-v^{1}} .
$$

Substituting (40) into (36) we arrive at the 
Lemma 3.1 (El \& Kamchatnov 2005 [7]): Hydrodynamic type system (36 ), (38) for $N=2$ reduces to a diagonal form in the field variables $v^{1}$ and $v^{2}$ :

$$
v_{t}^{1}=v^{2} v_{x}^{1}, \quad v_{t}^{2}=v^{1} v_{x}^{2}
$$

Remarkably, the hydrodynamic type system (41) is linearly degenerate because its characteristic velocities do not depend on the corresponding Riemann invariants. Physically this linear degeneracy reflects the already mentioned domination of the 'cross-component' soliton interactions over the interactions within a given component consisting of solitons with close amplitudes.

It is worth noting that system (41) is equivalent to the 1D Born-Infeld equation (Born \& Infeld 1934) arising in nonlinear electromagnetic field theory (see [42], [1])

$$
\left(1+\varphi_{x}^{2}\right) \varphi_{y y}-2 \varphi_{x} \varphi_{y} \varphi_{x y}+\left(1-\varphi_{y}^{2}\right) \varphi_{x x}=0
$$

As any two-component hydrodynamic type system, (41) is integrable (linearizable) via the classical hodograph transform. However, for any $N \geq 3$ integrability of the original system (36), (38) is no longer obvious. As a matter of fact, most $N$-component hydrodynamic type systems are not integrable for $N \geq 3$. Also, it is even not clear whether $N$-component system (36), (38) is linearly degenerate. It might seem that this system is simple enough for one to be able to verify these properties by a direct computation, using general definitions of linear degeneracy and integrability for hydrodynamic type systems [35], [39, 40] (also see the next Section). To our surprise, even the simplest non-trivial case $N=3$ turned out to be complicated enough to require computer algebra to get the confirmation of our hypothesis.

The identification of the system (36), (38) for $N=3$ as an integrable linearly degenerate hydrodynamic system can be considered as a strong indication that both properties (linear degeneracy and integrability) could hold true for this system for arbitrary $N$. Thus we formulate our main

Theorem $3.1 N$-component reductions (36), (38) of the generalized kinetic equation (1) are linearly degenerate integrable hydrodynamic type systems for any $N$.

To prove this theorem, we take advantage of the well-developed theory of integrable (semiHamiltonian) linearly degenerate hydrodynamic type systems [35], [11]. For convenience, in 
the next section we present a brief review of the main results of this theory which will be extensively used in Sections $5-8$ of the paper.

\section{Linearly degenerate integrable hydrodynamic type systems: account of properties}

A hydrodynamic type system

$$
U_{t}^{i}=v_{j}^{i}(\mathbf{U}) U_{x}^{j}, \quad i, j=1,2, \ldots, N
$$

is called semi-Hamiltonian (see [39, 40]) if it

(i) has $N$ mutually distinct eigenvalues $\lambda^{i}(\mathbf{U})$

$$
\operatorname{det}\left|\lambda \delta_{j}^{i}-v_{j}^{i}(\mathbf{U})\right|=0
$$

(ii) admits invertible point transformations $U^{k}(\mathbf{r})$, such that this hydrodynamic type system can be written in diagonal form

$$
r_{t}^{i}=V^{i}(\mathbf{r}) r_{x}^{i}, \quad i=1, \ldots, N
$$

The variables $r^{k}(\mathbf{U})$ are called Riemann invariants and $V^{k}(\mathbf{r})=\lambda^{k}(\mathbf{U}(\mathbf{r}))$ - characteristic velocities. Each Riemann invariant $r^{i}$ is determined up to an arbitrary function of a single variable $R_{i}\left(r^{i}\right)$.

(iii) satisfies the identity

$$
\partial_{j} \frac{\partial_{k} V^{i}}{V^{k}-V^{i}}=\partial_{k} \frac{\partial_{j} V^{i}}{V^{j}-V^{i}}, \quad i \neq j \neq k
$$

for each three distinct characteristic velocities $\left(\partial_{k} \equiv \partial / \partial r^{k}\right)$.

A semi-Hamiltonian hydrodynamic type system possesses infinitely many conservation laws parameterised by $N$ arbitrary functions of a single variable. Its general local solution for $\partial_{x} r^{i} \neq 0, i=1, \ldots, N$ is given by the generalised hodograph formula $[39,40]$

$$
x+V^{i}(\mathbf{r}) t=W^{i}(\mathbf{r}),
$$


where functions $W^{i}(\mathbf{r})$ are found from the linear system of PDEs:

$$
\frac{\partial_{i} W^{j}}{W^{i}-W^{j}}=\frac{\partial_{i} V^{j}}{V^{i}-V^{j}}, \quad i, j=1, \ldots, N, \quad i \neq j .
$$

Thus, the semi-Hamiltonian property (45) implies integrability of diagonal hydrodynamic type system in the above generalised hodograph sense.

It is known [40] that solutions $W^{j}$ of (47) specify commuting hydrodynamic flows to (44):

$$
r_{\tau}^{j}=W^{j}(\mathbf{r}) r_{x}^{j}, \quad j=1, \ldots, N
$$

where $\tau$ is a new time (group parameter). Indeed, one can readily show that equations (44), (48), (47) imply $\left(r_{\tau}^{j}\right)_{t}=\left(r_{t}^{j}\right)_{\tau}$.

A sub-class of linearly degenerate hydrodynamic type systems is distinguished by the property

$$
\partial_{i} V^{i}=0
$$

for each index $i$. It means that each characteristic velocity does not depend on the corresponding Riemann invariant $r^{i}$.

Theorem 4.1 (Pavlov 1987 [35]): If semi-Hamiltonian hydrodynamic type system (44) possesses conservation laws (36) with $u^{i}=U^{i}(\mathbf{r})$ and $v^{i}(\mathbf{U}(\mathbf{r}))=V^{i}(\mathbf{r})$ then this system is linearly degenerate. These conservation laws (36) are parameterized by $N$ arbitrary functions of a single variable.

Proof: The semi-Hamiltonian property (i.e. integrability) is given by the condition (45). We introduce, following Tsarev [40], the so-called Lame coefficients $\bar{H}_{i}$ by

$$
\partial_{k} \ln \bar{H}_{i}=\frac{\partial_{k} V^{i}}{V^{k}-V^{i}}, \quad i \neq k .
$$

Suppose that some semi-Hamiltonian hydrodynamic type system (44) can be written in the conservative form (36) with $v^{i}(\mathbf{U}(\mathbf{r}))=V^{i}(\mathbf{r})$. In such a case

$$
\partial_{k} U^{i} \cdot r_{t}^{k}=\partial_{k}\left(U^{i} V^{i}\right) \cdot r_{x}^{k}
$$

Since $\mathbf{r}(x, t)$ is an arbitrary solution of (44) we obtain $N$ equations

$$
V^{k} \cdot \partial_{k} U^{i}=\partial_{k}\left(U^{i} V^{i}\right)
$$


If $k \neq i$, then

$$
\partial_{k} \ln U^{i}=\frac{\partial_{k} V^{i}}{V^{k}-V^{i}}
$$

i.e. each of the conservation law densities $U^{i}$ is determined up to an arbitrary function of a single variable $P_{i}\left(r^{i}\right)$ (cf. (50) and (52)),

$$
U^{i}=\bar{H}_{i} \cdot P_{i}\left(r^{i}\right)
$$

If $k=i$, then it follows from (51) that $\partial_{i} V^{i}=0$ i.e. the system is linearly degenerate. The theorem is proved.

Remark 1: A subset $\left\{u^{k}\right\}$ of the conservation law densities $\left\{U^{k}\right\}$ satisfying a given system of conservation laws (e.g. (36), (38)) is selected by fixing the functions $P_{k}$ (e.g. $P_{k}\left(r^{k}\right) \equiv 1$ - see (99) in Section 7).

While converse of Theorem 4.1 is also true, one should note that not every conservation law of a semi-Hamiltonian linearly degenerate system satisfies the key property $v^{i}=V^{i}$. Indeed, let us consider the two-component system of conservation laws,

$$
U_{t}^{1}=\left(U^{1} v^{1}\left(U^{1}, U^{2}\right)\right)_{x}, \quad U_{t}^{2}=\left(U^{2} v^{2}\left(U^{1}, U^{2}\right)\right)_{x}
$$

Suppose this hydrodynamic type system is linearly degenerate, then it can be written in Riemann invariants $r^{1}\left(U^{1}, U^{2}\right), r^{2}\left(U^{1}, U^{2}\right)$ as follows:

$$
r_{t}^{1}=V^{1}\left(r^{1}, r^{2}\right) r_{x}^{1}, \quad r_{t}^{2}=V^{2}\left(r^{1}, r^{2}\right) r_{x}^{2}
$$

where $V^{1,2}(\mathbf{r})=v^{1,2}(\mathbf{U}(\mathbf{r}))$. Let us introduce new conservation law densities $\tilde{U}^{1}=U^{1}+U^{2}$ and $\tilde{U}^{2}=U^{1}-U^{2}$. Then the system of conservation laws (54) assumes an equivalent form

$$
\tilde{U}_{t}^{1}=\left(\tilde{U}^{1} \tilde{v}^{1}\left(\tilde{U}^{1}, \tilde{U}^{2}\right)\right)_{x}, \quad \tilde{U}_{t}^{2}=\left(\tilde{U}^{2} \tilde{v}^{2}\left(\tilde{U}^{1}, \tilde{U}^{2}\right)\right)_{x}
$$

where the characteristic velocities

$$
\tilde{v}^{1}=\frac{U^{1} v^{1}+U^{2} v^{2}}{U^{1}+U^{2}}, \quad \tilde{v}^{2}=\frac{U^{1} v^{1}-U^{2} v^{2}}{U^{1}-U^{2}}
$$

no longer coincide with $V^{1}\left(r^{1}, r^{2}\right)$ and $V^{2}\left(r^{1}, r^{2}\right)$. 
The full theory of linearly degenerate semi-Hamiltonian hydrodynamic type systems was constructed by Ferapontov in [11] using the Stäckel matrices

$$
\Delta=\left(\begin{array}{ccc}
\phi_{1}^{1}\left(r^{1}\right) & \ldots & \phi_{N}^{1}\left(r^{N}\right) \\
& & \\
\cdots & \cdots & \cdots \\
\phi_{1}^{N-2}\left(r^{1}\right) & & \phi_{N}^{N-2}\left(r^{N}\right) \\
\phi_{1}^{N-1}\left(r^{1}\right) & & \phi_{N}^{N-1}\left(r^{N}\right) \\
1 & \ldots & 1
\end{array}\right)
$$

where $\phi_{k}^{i}\left(r^{k}\right)$ are $N(N-1)$ arbitrary functions (it is clear that without loss of generality one can put $\phi_{k}^{N-1}(z)=z$ and the number of arbitrary function reduces to $\left.N(N-2)\right)$. Then characteristic velocities of such linearly degenerate hydrodynamic type systems are given by the formula

$$
V^{i}=\frac{\operatorname{det} \Delta_{i}^{(2)}}{\operatorname{det} \Delta_{i}^{(1)}},
$$

where $\Delta_{i}^{(k)}$ is the matrix $\Delta$ without $k$ th row and $i$ th column. The family of the conservation law densities $U^{i}$ corresponding to the semi-Hamiltonian system (44), (56) is determined by (cf. (53))

$$
U^{i}=\frac{\operatorname{det} \Delta_{i}^{(1)}}{\operatorname{det} \Delta}(-1)^{i+1} P_{i}\left(r^{i}\right)
$$

where $P_{i}\left(r^{i}\right), i=1, \ldots, N$ are arbitrary functions.

Corollary 4.1 The system of conservation laws (36) is a semi-Hamiltonian linearly degenerate hydrodynamic type system if and only if the densities $u^{i}$ and velocities $v^{i}(\mathbf{u})$ admit representations $u^{i}=U^{i}(\mathbf{r})$ and $v^{i}(\mathbf{U}(\mathbf{r}))=V^{i}(\mathbf{r})$, specified by (57), (56), via $N$ functions $r^{k}(x, t)$ satisfying diagonal system (44).

Proposition 4.1 (Ferapontov 1991 [11]): Semi-Hamiltonian linearly degenerate hydrodynamic type system (44), (56) has $N-2$ nontrivial linearly degenerate commuting flows

$$
r_{t^{k}}^{j}=V_{(k)}^{j}(\mathbf{r}) r_{x}^{j}, \quad j=1, \ldots, N, \quad k=3,4, \ldots, N
$$


whose characteristic velocities are determined as (cf. (56))

$$
V_{(k)}^{i}=\frac{\operatorname{det} \Delta_{i}^{(k)}}{\operatorname{det} \Delta_{i}^{(1)}} .
$$

Any characteristic velocity vector $\mathcal{W}(\mathbf{r})=\left(\mathcal{W}^{1}(\mathbf{r}), \mathcal{W}^{2}(\mathbf{r}), \ldots, \mathcal{W}^{N}(\mathbf{r})\right)$ specifying linearly degenerate hydrodynamic flow $r_{\tau}^{j}=\mathcal{W}^{j}(\mathbf{r}) r_{x}^{j}, j=1, \ldots, N$, commuting with (44), (56), can be represented as a linear combination of the "basis" characteristic velocity vectors $\mathbf{V}_{(k)}(59)$ (including "trivial" ones $\mathbf{V}_{(2)} \equiv \mathbf{V}$ (see $(56)$ ) and $\mathbf{V}_{(1)} \equiv \mathbf{1}$ ) with some constant coefficients $b_{k}$. Thus, for any component $\mathcal{W}^{i}$ there exists a decomposition

$$
\mathcal{W}^{i}=\sum_{k=1}^{N} b_{k} V_{(k)}^{i}
$$

Theorem 4.2 (Ferapontov 1991 [11]): General solution $\mathbf{r}(x, t)$ of the semi-Hamiltonian linearly degenerate system (44) is parameterized by $N$ arbitrary functions of one variable $f_{k}\left(r^{k}\right)$ and is given in an implicit form by the algebraic system

$$
\begin{aligned}
& x=\sum_{k=1}^{N} \int \frac{\phi_{k}^{1}(\xi) d \xi}{f_{k}(\xi)}, \quad-t=\sum_{k=1}^{N} \int \frac{r_{k}^{k}(\xi) d \xi}{f_{k}(\xi)} \\
& 0=\sum_{k=1}^{N} \int \frac{\phi_{k}^{M}(\xi) d \xi}{f_{k}(\xi)}, \quad M=3,4, \ldots, N .
\end{aligned}
$$

(note the change of sign for $t$ compared to [11] due to a slightly different representation of the diagonal system (44) in this paper). We also note that formulae (61) represent an equivalent of the symmetric generalised hodograph solution (46) for semi-Hamiltonian linearly degenerate hydrodynamic type systems.

It is instructive to introduce, following Darboux [10], the so-called rotation coefficients

$$
\beta_{i k}=\frac{\partial_{i} \bar{H}_{k}}{\bar{H}_{i}}, \quad i \neq k
$$

where the Lamé coefficients $\bar{H}_{i}$ are defined by (50). Then expression (45) for the semiHamiltonian property assumes the form of a Darboux system

$$
\partial_{i} \beta_{j k}=\beta_{j i} \beta_{i k}, \quad i \neq j \neq k
$$


Using (62) linear system (47) can be related to another linear system

$$
\partial_{i} H_{k}=\beta_{i k} H_{i}, \quad i \neq k
$$

via the so-called Combescure transformation (see [10])

$$
W^{i}=\frac{H_{i}}{\bar{H}_{i}}
$$

In other words, one can show (see [40]) that the ratio of any two solutions to (64) satisfies system (47) for the characteristic velocities of the commuting flows (48). We note that the particular solution $\tilde{H}_{i}$ of (64) corresponding to the characteristic velocities $V_{i}$ of the original system (44) is expressed in terms of the Lamé coefficient $\bar{H}_{i}$ as

$$
\tilde{H}_{i}=V^{i} \bar{H}_{i}
$$

Of course, general solution $H_{i}$ of system (64), as well as general solution $W^{i}$ of the generalized hodograph equations (47), is parameterized by $N$ arbitrary functions of a single variable.

Theorem 4.3 (Pavlov 1987 [35]): The class of the semi-Hamiltonian linearly degenerate systems of hydrodynamic type is selected, in addition to (63), by the set of restrictions on the rotation and Lame coefficients

$$
\partial_{i} \ln \bar{H}_{i}=\partial_{i} \ln \beta_{j i}
$$

for any index $j \neq i$.

Proof: Let us consider the Lamé coefficients for the linearly degenerate systems. Using (50), (49) we have

$$
\partial_{j} V^{i}=\partial_{j} \ln \bar{H}_{i} \cdot\left(V^{j}-V^{i}\right), \quad i \neq j, \quad \partial_{i} V^{i}=0 .
$$

The compatibility condition $\partial_{i}\left(\partial_{j} V^{i}\right)=\partial_{j}\left(\partial_{i} V^{i}\right)$ implies that

$$
\partial_{i} \partial_{j} \ln \bar{H}_{i}=\partial_{j} \ln \bar{H}_{i} \cdot \partial_{i} \ln \bar{H}_{j}, \quad i \neq j
$$

Now one can see that the 1.h.s. of (68) can be written in the form

$$
\partial_{i} \partial_{j} \ln \bar{H}_{i}=\partial_{i}\left(\frac{\bar{H}_{j}}{\bar{H}_{i}} \beta_{j i}\right)=\beta_{i j} \beta_{j i}+\frac{\bar{H}_{j}}{\bar{H}_{i}} \partial_{i} \beta_{j i}-\frac{\bar{H}_{j}}{\bar{H}_{i}^{2}} \beta_{j i} \partial_{i} \bar{H}_{i}
$$


On the other hand, the r.h.s. of (68) is nothing but the product $\beta_{i j} \beta_{j i}$. Now (67) immediately follows from (68) and (69). The Theorem is proved.

Now, suppose that the rotation coefficients (62) for some linearly degenerate hydrodynamic type system are given. Then restrictions (67) determine not only the Lamé coefficients (50) but also all other solutions of (64) associated, via (65), with the characteristic velocities (56), (59) of the complete set of linearly degenerate commuting flows. Indeed, one can see that equations (62), (67) actually represent $N$ systems of ordinary differential equations so that each system contains differentiation with respect to only one Riemann invariant. Thus, the general solution $\bar{H}_{i}$ of system (62), (67) is parameterized by $N$ arbitrary constants (see Proposition 4.1).

Let us introduce $N$ particular solutions $\bar{H}_{i}^{(k)}$ of system (62), (67) such that (see (56), (59))

$$
V_{(k)}^{i}=\frac{\bar{H}_{i}^{(k)}}{\bar{H}_{i}}, \quad k=1,2, \ldots, N,
$$

where $\bar{H}_{i}=\bar{H}_{i}^{(1)}, \tilde{H}_{i}=\bar{H}_{i}^{(2)}$ (see (59)). As a matter of fact, $V_{(2)}^{i} \equiv V^{i}, V_{(1)}^{i} \equiv 1$. Then (67) can be written in a slightly more general form,

$$
\partial_{i} \ln \beta_{j i}=\partial_{i} \ln \bar{H}_{i}^{(k)}
$$

- for any $k$ and $j \neq i$.

Thus, the full class of linearly degenerate semi-Hamiltonian hydrodynamic type systems is determined by conditions (67), (62) and (63). We note that system (67), (62) and (63) is an overdetermined system in involution. Its integration leads to the aforementioned set of particular solutions of (64) that can be parameterized via a Stäckel matrix (see (55), (56), (57) and (59)).

\section{$5 \quad N=3$ : explicit formulae}

We now consider the first nontrivial (from the viewpoint of integrability) case $N=3$ of the hydrodynamic reduction (36), (38). To prove our main Theorem 3.1 for $N=3$ we shall make use of Corollary 4.1. 
Let us suppose that hydrodynamic system of conservation laws (36), (38) is linearly degenerate and can be written in a diagonal form (44), i.e. we suppose that there exists an invertible change of variables $r^{j}(\mathbf{u}), j=1,2,3$, such that system (36) assumes a diagonal form

$$
r_{t}^{j}=V^{j}(\mathbf{r}) r_{x}^{j}, \quad j=1,2,3
$$

where $V^{j}(\mathbf{r})=v^{j}(\mathbf{u}(\mathbf{r}))$.

We introduce the Stäckel matrix (55), which for $N=3$ can be written in the form

$$
\Delta=\left(\begin{array}{ccc}
B_{1}\left(r^{1}\right) & B_{2}\left(r^{2}\right) & B_{3}\left(r^{3}\right) \\
A_{1}\left(r^{1}\right) & A_{2}\left(r^{2}\right) & A_{3}\left(r^{3}\right) \\
1 & 1 & 1
\end{array}\right),
$$

where $A_{k}(z)$ and $B_{k}(z)$ are arbitrary functions.

Now, by Corollary 4.1, if system (36), (38) is linearly degenerate and semi-Hamiltonian then its diagonal representation (70) must have characteristic velocities in the form (56), i.e. for $N=3$ we have

$$
V^{1}=\frac{B_{2}\left(r^{2}\right)-B_{3}\left(r^{3}\right)}{A_{2}\left(r^{2}\right)-A_{3}\left(r^{3}\right)}, \quad V^{2}=\frac{B_{3}\left(r^{3}\right)-B_{1}\left(r^{1}\right)}{A_{3}\left(r^{3}\right)-A_{1}\left(r^{1}\right)}, \quad V^{3}=\frac{B_{1}\left(r^{1}\right)-B_{2}\left(r^{2}\right)}{A_{1}\left(r^{1}\right)-A_{2}\left(r^{2}\right)} .
$$

Then, using (57) the corresponding conservation law densities $u^{k}$ are found in terms of Riemann invariants as

$$
u^{1}=\frac{P_{1}\left(r^{1}\right)}{\operatorname{det} \Delta}\left[A_{2}\left(r^{2}\right)-A_{3}\left(r^{3}\right)\right], \quad u^{2}=\frac{P_{2}\left(r^{2}\right)}{\operatorname{det} \Delta}\left[A_{3}\left(r^{3}\right)-A_{1}\left(r^{1}\right)\right], \quad u^{3}=\frac{P_{3}\left(r^{3}\right)}{\operatorname{det} \Delta}\left[A_{1}\left(r^{1}\right)-A_{2}\left(r^{2}\right)\right],
$$

where $P_{j}\left(r^{j}\right)$ are arbitrary functions and the determinant of the Stäckel matrix is given by

$$
\operatorname{det} \Delta=A_{1}\left(r^{1}\right)\left[B_{2}\left(r^{2}\right)-B_{3}\left(r^{3}\right)\right]+A_{2}\left(r^{2}\right)\left[B_{3}\left(r^{3}\right)-B_{1}\left(r^{1}\right)\right]+A_{3}\left(r^{3}\right)\left[B_{1}\left(r^{1}\right)-B_{2}\left(r^{2}\right)\right]
$$

Substitution of (72)-(74) into (38) yields expressions for the functions $A_{k}(z), B_{k}(z)$, $P_{k}(z), k=1,2,3$.

Before we present these expressions, we note that it follows from (72), (74) that functions $B_{k}(z)$ are determined up to a constant shift which is then translated into a certain shift for the functions $P_{k}(z)$. It turns out that this shift can be removed by the simplest change of 
the Riemann invariants, $\left(r^{k}+\right.$ constant) $\mapsto r^{k}$ (although the relationships between the shift constants for $B_{k}, P_{k}$ and $r^{k}$ are rather cumbersome) so that we eventually obtain

$$
A_{i}\left(r^{i}\right)=r^{i}, \quad B_{i}\left(r^{i}\right)=\zeta_{i} r^{i}, \quad i=1,2,3
$$

where

$$
\begin{gathered}
\zeta_{1}=\frac{\xi_{3} \epsilon_{12}-\xi_{2} \epsilon_{13}}{\epsilon_{12}-\epsilon_{13}}, \quad \zeta_{2}=\frac{\xi_{1} \epsilon_{23}-\xi_{3} \epsilon_{12}}{\epsilon_{23}-\epsilon_{12}}, \quad \zeta_{3}=\frac{\xi_{1} \epsilon_{23}-\xi_{2} \epsilon_{13}}{\epsilon_{23}-\epsilon_{13}} \\
P_{1}=\frac{\xi_{2}-\xi_{3}}{\epsilon_{12}-\epsilon_{13}} r^{1}+\epsilon_{23}, \quad P_{2}=\frac{\xi_{1}-\xi_{3}}{\epsilon_{12}-\epsilon_{23}} r^{2}+\epsilon_{13}, \quad P_{3}=\frac{\xi_{1}-\xi_{2}}{\epsilon_{13}-\epsilon_{23}} r^{3}+\epsilon_{12} .
\end{gathered}
$$

Direct verification shows that the diagonal system (70), (72), (75), (76) is indeed equivalent, via $(73),(74),(77)$, to the original set of conservation laws (36), (38), where $v^{k}(\mathbf{u}(\mathbf{r}))=$ $V^{k}(\mathbf{r})$.

Thus, system (36), (38) is consistent with formulae (72), (73) defined by the Stäckel matrix (71). Therefore, by Corollary 4.1, the three-component hydrodynamic reduction (36), (38) is a linearly degenerate semi-Hamiltonian (i.e. integrable) hydrodynamic type system.

Remark. As we have seen, the outlined construction has some additional inherent "degrees of freedom", namely, three arbitrary constants due to non-uniqueness of the Stäckel matrix specifying a given linearly degenerate semi-Hamiltonian system. The full set of arbitrary constants removable by an appropriate change of the Riemann invariants will appear later in Section 5 where we shall consider $N$-component hydrodynamic reductions with arbitrary $N \geq 3$.

Using (72)-(77) we obtain explicit expressions for the characteristic velocities $V^{k}$ and densities $u^{k}$ in terms of Riemann invariants,

$$
\begin{aligned}
V^{1} & =\frac{\zeta_{2} r^{2}-\zeta_{3} r^{3}}{r^{2}-r^{3}}, \quad V^{2}=\frac{\zeta_{3} r^{3}-\zeta_{1} r^{1}}{r^{3}-r^{1}}, \quad V^{3}=\frac{\zeta_{1} r^{1}-\zeta_{2} r^{2}}{r^{1}-r^{2}}, \\
u^{1} & =P_{1} \frac{r^{2}-r^{3}}{\operatorname{det} \Delta}, \quad u^{2}=P_{2} \frac{r^{3}-r^{1}}{\operatorname{det} \Delta}, \quad u^{3}=P_{3} \frac{r^{1}-r^{2}}{\operatorname{det} \Delta},
\end{aligned}
$$

where

$$
\operatorname{det} \Delta=\left(\zeta_{1}-\zeta_{2}\right) r^{1} r^{2}+\left(\zeta_{2}-\zeta_{3}\right) r^{2} r^{3}+\left(\zeta_{3}-\zeta_{1}\right) r^{3} r^{1}
$$


We note that, unlike in the case $N=2$, algebraic system (38) cannot be resolved for $u^{k}$ in terms of $v^{n}$ for any odd $N$ (cf. corresponding formulae in Section 2), because determinant of the matrix $\hat{\mathbf{A}}$ of linear system (38)

$$
\hat{\mathbf{A}} \mathbf{u}=\mathbf{b},
$$

where $A_{i k}=\epsilon_{i k}\left(v^{k}-v^{i}\right)$ and $b_{i}=v^{i}-\xi_{i}$, equals zero due to its skewsymmetry. For instance, for $N=3$, the consistency condition of this linear system (i.e. the condition that the rank of augmented matrix equals 2) is given by the relation

$$
\epsilon_{23}\left(v^{3}-v^{2}\right)\left(\xi_{1}-v^{1}\right)+\epsilon_{12}\left(v^{2}-v^{1}\right)\left(\xi_{3}-v^{3}\right)+\epsilon_{13}\left(v^{1}-v^{3}\right)\left(\xi_{2}-v^{2}\right)=0 .
$$

Direct substitution of $v^{j}=V^{j}(\mathbf{r})$ (78) into (81) shows that it satisfies identically.

Using (79), (80), (76), (77) one can express the Riemann invariants in terms of the densities $u^{k}$ explicitly,

$$
\begin{aligned}
r^{1} & =\frac{\left(\epsilon_{12}-\epsilon_{13}\right)\left(\epsilon_{12} \epsilon_{13} u^{1}+\epsilon_{12} \epsilon_{23} u^{2}+\epsilon_{13} \epsilon_{23} u^{3}+\epsilon_{23}\right)}{\left[\left(\xi_{3}-\xi_{1}\right) \epsilon_{12}+\left(\xi_{1}-\xi_{2}\right) \epsilon_{13}\right] u^{1}-\left(\xi_{2}-\xi_{3}\right)\left(\epsilon_{12} u^{2}+\epsilon_{13} u^{3}+1\right)}, \\
r^{2} & =\frac{\left(\epsilon_{23}-\epsilon_{12}\right)\left(\epsilon_{12} \epsilon_{13} u^{1}+\epsilon_{12} \epsilon_{23} u^{2}+\epsilon_{13} \epsilon_{23} u^{3}+\epsilon_{13}\right)}{\left[\left(\xi_{1}-\xi_{2}\right) \epsilon_{23}+\left(\xi_{2}-\xi_{3}\right) \epsilon_{12}\right] u^{2}-\left(\xi_{3}-\xi_{1}\right)\left(\epsilon_{12} u^{1}+\epsilon_{23} u^{3}+1\right)}, \\
r^{3} & =\frac{\left(\epsilon_{13}-\epsilon_{23}\right)\left(\epsilon_{12} \epsilon_{13} u^{1}+\epsilon_{12} \epsilon_{23} u^{2}+\epsilon_{13} \epsilon_{23} u^{3}+\epsilon_{12}\right)}{\left[\left(\xi_{2}-\xi_{3}\right) \epsilon_{13}+\left(\xi_{3}-\xi_{1}\right) \epsilon_{23}\right] u^{3}-\left(\xi_{1}-\xi_{2}\right)\left(\epsilon_{13} u^{1}+\epsilon_{23} u^{2}+1\right)} .
\end{aligned}
$$

Direct substitution shows that expressions (82) and (78) are consistent with original algebraic system (38) where $v^{j}=V^{j}(\mathbf{r}(\mathbf{u}))$.

It is instructive to look at what happens to the diagonal system (70) when the density of one of the components in conservation laws (36), say $u^{3}$, vanishes. One can see from (82) that if $u^{3}=0$ (this corresponds to vanishing of $P_{3}$ in (79)) then the Riemann invariant $r^{3}$ becomes a constant,

$$
u^{3}=0: \quad r^{3}=-\frac{\left(\epsilon_{23}-\epsilon_{13}\right) \epsilon_{12}}{\xi_{1}-\xi_{2}} \equiv r_{0}^{3},
$$

so that the equation for $r^{3}$ satisfies identically and system (70) reduces to its 2 -component counterpart (41) for

$$
v^{1}\left(u^{1}, u^{2}\right)=V^{1}\left(r^{2}\left(u^{1}, u^{2}, 0\right)\right), \quad v^{2}\left(u^{1}, u^{2}\right)=V^{2}\left(r^{1}\left(u^{1}, u^{2}, 0\right)\right),
$$


as one should expect. Similar reductions occur for $u^{1}=0$ and $u^{2}=0$, which lead to $r^{1}=r_{0}^{1}=$ constant and $r^{2}=r_{0}^{2}=$ constant respectively. As a matter of fact, any function $R^{j}\left(r^{j}\right)$ is also a Riemann invariant so one can choose a new set of Riemann invariants say $R^{j}=r^{j}-r_{0}^{j}$ so that $R^{j}=0$ when $u^{j}=0$. This normalisation could be useful for applications.

Now we consider some special families of solutions to linearly degenerate system (70), $(78)$.

\section{a) Similarity solutions}

One can see that, owing to homogeneity of the characteristic velocities (78) as functions of Riemann invariants, system (70) admits similarity solutions of the form

$$
r^{i}=\frac{1}{t^{\alpha}} l^{i}\left(\frac{x}{t}\right), \quad i=1,2,3,
$$

where $\alpha$ is an arbitrary positive real number and the functions $l^{i}(\tau)$, where $\tau=x / t$, satisfy the system of ordinary differential equations

$$
\left(V^{i}(\mathbf{l})+\tau\right) \frac{d l^{i}}{d \tau}+\alpha l^{i}=0, \quad i=1,2,3
$$

Here the functions $V^{i}(\mathbf{l})$ are obtained from (78) by replacing $r^{i}$ with $l^{i}$. It is not difficult to see that, due to the structure of the characteristic velocities, the case $\alpha=0$ implies only a constant solution $l^{i}=l_{0}^{i}$, where $l_{0}^{1}, l_{0}^{2}, l_{0}^{3}$ are arbitrary constants. If $\alpha \neq 0$, the general solution of (84) can be found in an implicit form using the generalised hodograph formulae (61), where for $N=3$ we substitute, according to $(71),(75), \phi_{k}^{1}(\xi) \equiv B_{k}(\xi)=\zeta_{k} \xi, \phi_{k}^{2}(\xi) \equiv A_{k}(\xi)=\xi$. To obtain similarity solutions (83) one should use in (61) $f_{i}(\xi)=\xi^{\beta} / c_{i}$, where $\beta=2+1 / \alpha$ and $c_{i}, i=1,2,3$, are arbitrary nonzero constants. Then the requirement that the functions $l^{i}$ must depend on $\tau=x / t$ alone leads to the algebraic system

$$
\begin{aligned}
\tau & =c_{1} \zeta_{1}\left(l^{1}\right)^{\gamma}+c_{2} \zeta_{2}\left(l^{2}\right)^{\gamma}+c_{3} \zeta_{3}\left(l^{3}\right)^{\gamma}, \\
-1 & =c_{1}\left(l^{1}\right)^{\gamma}+c_{2}\left(l^{2}\right)^{\gamma}+c_{3}\left(l^{3}\right)^{\gamma}, \\
0 & =c_{1}\left(l^{1}\right)^{\gamma-1}+c_{2}\left(l^{2}\right)^{\gamma-1}+c_{3}\left(l^{3}\right)^{\gamma-1},
\end{aligned}
$$


where $\gamma=-1 / \alpha$ and we have also replaced $c_{i} / \gamma \mapsto c_{i}$. Direct substitution shows that solution $l^{i}$ defined by (85) indeed satisfies system (84). We note that this family of solutions is unique to the case $N=3$ and generally does not exist for $N>3$.

\section{b) Quasiperiodic solutions}

Another interesting type of solutions arises when one introduces in (61) (for $N=3$ )

$$
f_{1}(\xi)=f_{2}(\xi)=f_{3}(\xi)=\sqrt{R_{7}(\xi)}, \quad R_{7}(\xi)=\prod_{n=1}^{7}\left(\xi-E_{n}\right),
$$

where $E_{1}<E_{2}<\cdots<E_{7}$ are real constants. Then, according to (75), solution (61) assumes the form

$$
\begin{aligned}
x & =\zeta_{1} \int \frac{\xi d \xi}{\sqrt{R_{7}(\xi)}}+\zeta_{2} \int \frac{\xi d \xi}{\sqrt{R_{7}(\xi)}}+\zeta_{3} \int \frac{r^{3}}{\sqrt{R_{7}(\xi)}}, \\
-t & =\int \frac{\xi d \xi}{\sqrt{R_{7}(\xi)}}+\int \frac{\xi d \xi}{\sqrt{R_{7}(\xi)}}+\int \frac{\xi d \xi}{\sqrt{R_{7}(\xi)}}, \\
0 & =\int \frac{r^{2}}{\sqrt{R_{7}(\xi)}}+\int \frac{d \xi}{\sqrt{R_{7}(\xi)}}+\int \frac{d \xi}{\sqrt{R_{7}(\xi)}},
\end{aligned}
$$

which resembles the celebrated system for the multi-gap (here - three-gap) solutions of the $\mathrm{KdV}$ equation. Unlike (86) - (88), however, the three-gap KdV solutions correspond to the Stäckel matrix (71) with the rows $A_{k}(\xi)=\xi, B_{k}(\xi)=\xi^{2}, k=1,2,3[11]$.

Proposition 5.1. For any constants $\zeta_{1} \neq \zeta_{2} \neq \zeta_{3} \neq 0$ there exists at least one set $\left\{E_{1}, \ldots, E_{6}\right\}$ such that the solution $r^{i}(x, t), i=1,2,3$ described by (86) - (88) is quasiperiodic in $x$ and possibly in $t$.

We present here a sketch of the proof. Availability of the solution in the form (86) (88) implies the existence of separate dynamics of $r^{j}$-s with respect to $x$ and $t$. Indeed, differentiating (86) - (88) with respect to $x$ for fixed $t$ one readily obtains

$$
\frac{\partial r^{i}}{\partial x}=\left(r^{j}-r^{k}\right) \frac{\sqrt{R_{7}\left(r_{i}\right)}}{\Pi}, \quad i, j, k=1,2,3, \quad i \neq j \neq k
$$

where

$$
\Pi\left(r_{1}, r_{2}, r_{3}\right)=\left(\zeta_{1}-\zeta_{2}\right) r^{1} r^{2}+\left(\zeta_{2}-\zeta_{3}\right) r^{2} r^{3}+\left(\zeta_{3}-\zeta_{1}\right) r^{3} r^{1}=\operatorname{det} \Delta
$$


- see $(80)$.

Analogously, differentiating (86) - (88) with respect to $t$ for fixed $x$ one obtains

$$
\frac{\partial r^{i}}{\partial t}=\left(\zeta_{j} r^{j}-\zeta_{k} r^{k}\right) \frac{\sqrt{R_{7}\left(r_{i}\right)}}{\Pi}, \quad i, j, k=1,2,3, \quad i \neq j \neq k .
$$

One can see that the flows (89) and (91) are consistent with the spatio-temporal dynamics (44), (78). We also note that equations (89), (91) resemble Dubrovin's equations for the auxiliary spectrum dynamics in the KdV finite-gap integration problem (see, for instance, [33]).

Let us now suppose that

$$
r^{1} \in\left[E_{1}, E_{2}\right], r^{2} \in\left[E_{3}, E_{4}\right], r^{3} \in\left[E_{5}, E_{6}\right],
$$

so that all $\sqrt{R_{7}\left(r^{i}\right)}$ are real. The above condition (92) means that the point $p=\left(r^{1}, r^{2}, r^{3}\right) \in$ $\mathbb{R}^{3}$ lies within the rectangular box $K_{i j k} \in \mathbb{R}^{3}$ with the vertices at $\left(E_{i}, E_{j}, E_{k}\right), i, j, k=$ $1, \ldots, 6, i \neq j \neq k$.

Now, for any set of the constants $\zeta_{1}, \zeta_{2}, \zeta_{3}$ there exists at least one box $K_{i, j, k}=K^{*} \in \mathbb{R}^{3}$, which is not intersected by the cone $\Pi\left(r^{1}, r^{2}, r^{3}\right)=0$. That is, inside $K^{*}$ the denominator $\Pi\left(r_{1}, r_{2}, r_{3}\right)$ in (89) never vanishes.

Assume now that the 'initial' values of $r^{1}, r^{2}, r^{3}$ for some $x=x_{0}$ belong to $K^{*}$. Then it follows from (89) that, under the $x$-flow $\left(t=\right.$ const), the point $p$ remains inside $K^{*}$ and undergoes "elastic" reflections at the faces of $K^{*}$ as $x$ varies (note that, since $r^{j} \neq r^{k}$ for $j \neq k$, the factor $\left(r^{j}-r^{k}\right)$ in (89) never vanishes so the reflections occur only at the faces of $K^{*}$ ). Therefore, the motion is quasi-periodic with respect to $x$ as long as conditions (92) are satisfied. Indeed, the system (89) possesses two integrals (87) and (88) outside the "resonant" points, where $\Pi=0$, so it specifies a quasi-periodic motion on a 3 -torus provided conditions (92) are satisfied. Of course, if conditions (92) are not satisfied at $x=x_{0}$ the solutions $r^{i}(x)$ may blow up and not be quasi-periodic.

The proof of quasi-periodicity of the $t$-flow is similar, however, there is an additional requirement that the factor $\left(\zeta_{j} r^{j}-\zeta_{k} r^{k}\right)$ in (90) should not vanish for all $\mathbf{r} \in K^{*}$ which might impose additional restrictions on the choice of $E_{i}$ (that is for some $\left\{E_{j}\right\}$ the motion can be quasi-periodic in $x$ but not in $t$ ). 
We note that the quasi-periodicity of the $x$ - and $t$-flows can be proved directly from the solution (86) - (88), however the outlined proof using the dynamical systems arguments is qualitatively more transparent and more readily yields the "resonant" restrictions for $x$ - and $t$-flows. We also note that the quasiperiodic solutions could be constructed for $N>3$ as well (see Section 8.2).

\section{Integrability of $N$-component hydrodynamic reduc- tions}

We now prove our main Theorem 3.1 stating that the $N$-component 'cold-gas' hydrodynamic reduction (36), (38) represents a semi-Hamiltonian (i.e. integrable) linearly degenerate hydrodynamic type system. For that, according to Corollary 3.1, it is sufficient to show that the conservation law densities $u^{i}$ and the transport velocities $v^{i}$ admit parametric representations (57) and (56), $u^{i}=U^{i}(\mathbf{r})$ and $v^{i}(\mathbf{U}(\mathbf{r}))=V^{i}(\mathbf{r})$, via $N$ functions $r^{k}$ in terms of the Stäckel matrix (55).

We suppose that hydrodynamic type system (36), (38) can be rewritten in a diagonal form (44), and, moreover, the characteristic velocities $V^{i}(\mathbf{r})$ coincide with the expressions $v^{i}(\mathbf{U}(\mathbf{r}))$.

Now, substitution of (56), (57) into (38) leads to the algebraic system

$$
\sum_{k=1}^{N} \epsilon_{i k}(-1)^{k} P_{k} \operatorname{det} \Delta_{i k}^{(12)}=\operatorname{det} \Delta_{i}^{(2)}-\xi_{i} \operatorname{det} \Delta_{i}^{(1)}, \quad i=1, \ldots, N
$$

for $P_{k}\left(r^{k}\right)$ and $\phi_{k}^{i}\left(r^{k}\right)$. Here the matrix $\Delta_{i k}^{(12)}$ is the matrix $\Delta$ with first two rows and $i$ th and $k$ th columns deleted. In the derivation of (93) we have used the determinant Sylvester identity (see, for instance, Gantmaher 1959)

$$
\operatorname{det} \Delta_{i k}^{(12)}=\frac{\operatorname{det} \Delta_{k}^{(1)} \operatorname{det} \Delta_{i}^{(2)}-\operatorname{det} \Delta_{i}^{(1)} \operatorname{det} \Delta_{k}^{(2)}}{\operatorname{det} \Delta}
$$

Expanding the determinants,

$$
\operatorname{det} \Delta_{i}^{(1)}=\sum_{k=1}^{N}\left[(-1)^{k+1} \phi_{k}^{2} \operatorname{det} \Delta_{i k}^{(12)}\right], \quad \operatorname{det} \Delta_{i}^{(2)}=\sum_{k=1}^{N}\left[(-1)^{k+1} \phi_{k}^{1} \operatorname{det} \Delta_{i k}^{(12)}\right],
$$


we rewrite equations (93) as $N$ nonlinear systems for $\phi_{k}^{n}$ and $P_{k}$, where $k, n=1, \ldots, N$,

$$
\sum_{k=1}^{N}(-1)^{k}\left(\phi_{k}^{1}-\xi_{i} \phi_{k}^{2}+\epsilon_{i k} P_{k}\right) \operatorname{det} \Delta_{i k}^{(12)}=0, \quad i=1, \ldots, N
$$

We recall that $\phi_{k}^{N-1}=r^{k}, \phi_{k}^{N}=1$.

One can now introduce $N$ matrices $\delta_{i}$ obtained from the matrix $\Delta$ by deleting the first two rows and the $i$-th column, and adding the first row with the elements $\phi_{k}^{1}-\xi_{i} \phi_{k}^{2}+\epsilon_{i k} P_{k}$. Thus, each matrix $\delta_{i}$ has dimension $(N-1) \times(N-1)$. Then the above set of equations (94) can be rewritten as

$$
\operatorname{det} \delta_{i}=0, \quad i=1, \ldots, N
$$

which implies that the rows of each of the matrices $\delta_{i}$ must be linearly dependent:

$$
\begin{aligned}
C_{i, 1}\left(\epsilon_{i k} P_{k}+\phi_{k}^{1}-\xi_{i} \phi_{k}^{2}\right)+\sum_{n=3}^{N-2} C_{i, n-1} \phi_{k}^{n} & =C_{i, N-2} r^{k}+C_{i, N-1}, \\
k & =1, \ldots N, \quad i=1, \ldots, N-1, \quad k \neq i,
\end{aligned}
$$

where $C_{i, k}$ are arbitrary constants. These conditions can be considered as $N$ linear systems, for fixed $k$ each. Since all these systems are consistent the functions $\phi_{k}^{i}$ and $P_{k}$ can be found by solving system (96).

Constants $C_{i, 1}$ cannot be equal to zero since in that case, according to (56), the velocities $V^{i}$ would become undetermined. Therefore, without loss of generality we can set $C_{i, 1}=1$ and the number of free constants becomes $N(N-2)$. Thus, the following Proposition is valid:

Proposition 6.1: General solution of system (94) is determined by solutions

$$
\phi_{k}^{i}=\frac{\operatorname{det} \tilde{B}_{k}^{i} r^{k}+\operatorname{det} \bar{B}_{k}^{i}}{\operatorname{det} B_{k}}, \quad P_{k}=\frac{\operatorname{det} B_{k}^{(P)}}{\operatorname{det} B_{k}}
$$

of $N$ linear systems (96), where $B_{k}, \bar{B}_{k}^{i}, \tilde{B}_{k}^{i}$ and $B_{k}^{(P)}$ are matrices with elements

$$
\tilde{b}_{k l}^{i m}=\bar{b}_{k l}^{i m}=b_{k l}^{(P) m}=b_{k l}^{m}=\left\{\begin{array}{ccc}
1 & \text { for } l=2 \\
-\xi_{l} & \text { for } l=3 \\
C_{m, l-2} & \text { for } l>3
\end{array} \quad \text { if } \quad l \neq i+1\right.
$$

and $b_{k 1}^{i m}=\tilde{b}_{k 1}^{i m}=\bar{b}_{k 1}^{i m}=\epsilon_{m k}, \bar{b}_{k i}^{i+1 m}=C_{i, N-1}, \tilde{b}_{k i}^{i+1 m}=C_{i, N-2}, b_{k 1}^{(P) m}=C_{i, N-2} r^{k}+C_{i, N-1}$, 
where $C_{m, l}$ are arbitrary constants such that $\operatorname{det} B_{k} \neq 0$.

Remark: The set of constants $C_{l, m}$ for which $\operatorname{det} B_{k}=0$ has Lebesque measure zero or requires a very special choice of the parameters $\eta_{k}$. The exceptional case is the following: the vectors $\xi, \mathbf{1}$ and $\epsilon_{k}$ are linearly dependent which yields, according to the definition (39), a set of equations for the special values $\eta_{k}$.

Thus, we have proved that all elements of the Stäckel matrix (55) depend linearly on Riemann invariants and these elements are determined from the algebraic system (38) up to $N(N-2)$ arbitrary constants removable by an appropriate change of the Riemann invariants (for instance, by a shift in the case $N=3$ ). By Corollary 4.1, the existence of such a Stäckel matrix automatically proves the semi-Hamiltonian and linearly-degenerate properties of the hydrodynamic reductions (36), (38).

Now, our main Theorem 3.1 is proved.

\section{Riemann invariants and characteristic velocities: ex- plicit construction}

The construction described in Sections 3 and 6 provides a proof of the existence of Riemann invariants for system (36), (38) for arbitrary $N$. The Riemann invariants are found to parameterize system (36), (38) via the sole Stäckel matrix, which, by Corollary 4.1, implies linear degeneracy and integrability of this system. Explicit representations for conservation law densities $u^{i}$ and transport velocities $v^{j}$ in terms of the Riemann invariants are given by Ferapontov [11] formulae (57), (56) where the entries $\phi_{k}^{n}$ of the Stäckel matrix (55) and the functions $P_{k}\left(r^{k}\right)$ are defined by formulae $(97)-(98)$. Using the functions $\phi_{k}^{n}$ one also obtains the generalised hodograph solutions (61).

The outlined procedure, while providing general theoretical framework for the study of the 'cold-gas' reductions of the kinetic equation for a soliton gas, seems to be not very convenient from the viewpoint of practical calculations. It also involves $N(N-2)$ intermediate constants $C_{l, m}$, which introduce an additional unnecessary complication. It is, thus, desirable to have 
more direct representations for the Riemann invariants and characteristic velocities, which will also be free from these intermediate arbitrary constants.

We shall make use of the Theorem 3.1 and show that, once the linear degeneracy and integrability properties of system (36), (38) are established, explicit relations between the Riemann invariants $\mathbf{r}$ and the conserved densities $\mathbf{u}$ can be found by a relatively straightforward calculation. The calculation will involve the properties of the Lamé coefficients outlined in Section 4.

First, without loss of generality we choose the following normalization (see (53))

$$
u^{k}=\bar{H}_{k}
$$

where $\bar{H}_{k}$ 's are the Lamé coefficients (50). Now, using Theorem 3.1 we assume that hydrodynamic type system (36), (38) can be rewritten in a diagonal form (44), so that $u^{i}=U^{i}(\mathbf{r})$ and $v^{i}(\mathbf{U}(\mathbf{r}))=V^{i}(\mathbf{r})$. For convenience, in what follows we shall use small $u$ 's and $v$ 's only, assuming that $u_{j}=u_{j}(\mathbf{r}) \equiv U^{j}(\mathbf{r}), v_{j}=v_{j}(\mathbf{r}) \equiv V_{j}(\mathbf{r})$.

To obtain explicit formulae for the Riemann invariants of the hydrodynamic reduction (36), (38) we need first to prove its so-called "Egorov" property.

Definition 7.1 (Pavlov \& Tsarev 2003 [38]): Semi-Hamiltonian hydrodynamic type system (42) is called the Egorov, if a sole pair of conservation laws

$$
\partial_{t} a(\mathbf{u})=\partial_{x} b(\mathbf{u}), \quad \partial_{t} b(\mathbf{u})=\partial_{x} c(\mathbf{u})
$$

exists.

It was proved in [38], that

$$
\partial_{i} a=\bar{H}_{i}^{2}, \quad \partial_{i} b=\tilde{H}_{i} \bar{H}_{i}, \quad \partial_{i} c=\tilde{H}_{i}^{2},
$$

(see $(50)$ and $(66)$ for the definitions of $\bar{H}_{i}$ and $\tilde{H}_{i}$ ) while the corresponding rotation coefficients (62) become symmetric, i.e.

$$
\beta_{i k}=\beta_{k i}, \quad i \neq k
$$

Another important fact proven in [38] is that all commuting flows to a semi-Hamiltonian Egorov system are also Egorov so commuting flow (48) possesses a similar pair of conservation 
laws

$$
\partial_{\tau} a(\mathbf{u})=\partial_{x} h(\mathbf{u}), \quad \partial_{\tau} h(\mathbf{u})=\partial_{x} g(\mathbf{u})
$$

where

$$
\partial_{i} h=H_{i} \bar{H}_{i}, \quad \partial_{i} g=H_{i}^{2}
$$

Now we prove the following

Lemma 7.1: Hydrodynamic reductions (36), (38) are Egorov.

Proof: We consider the sum of conservation laws (36), (38)

$$
\partial_{t}\left(\sum u^{k}\right)=\partial_{x}\left(\sum u^{k} v^{k}\right)=\partial_{x}\left[\sum_{k=1}^{N} u^{k}\left(\xi_{k}+\sum_{m \neq k} \epsilon_{k m} u^{m}\left(v^{m}-v^{k}\right)\right)\right]
$$

One can see that, since the matrix $\epsilon_{i k}$ is symmetric, the last term in r.h.s. of (104) vanishes. Thus, (104) simplifies to the form

$$
\partial_{t}\left(\sum u^{k}\right)=\partial_{x}\left(\sum \xi_{k} u^{k}\right)
$$

However, the flux $\Sigma \xi_{k} u^{k}$ of conservation law (105) is nothing but the density of another conservation law which can be obtained by the same summation but with the special weights $\xi_{i}$, i.e.

$$
\partial_{t}\left(\sum \xi_{k} u^{k}\right)=\partial_{x}\left(\sum \xi_{k} u^{k} v^{k}\right)
$$

Comparison with definition (100) implies that in our case

$$
a=\sum u^{m}, \quad b=\sum \xi_{m} u^{m} \equiv \sum u^{m} v^{m}, \quad c=\sum \xi_{m} u^{m} v^{m}
$$

which completes the proof.

Now we formulate the following

Theorem 7.1: The Riemann invariants of $N$-component hydrodynamic reductions (36), (38) can be found explicitly as

$$
r^{i}=-\frac{1}{u^{i}}\left(1+\sum_{m \neq i} \epsilon_{i m} u^{m}\right), \quad i=1, \ldots, N .
$$

Proof: 
For the sake of completeness of our construction we first show that the linear degeneracy property (49) of system (36), (38) readily follows from the (already established) existence of the Riemann invariants $r^{k}$. Indeed, differentiating (38) with respect to the Riemann invariant $r^{i}$ and taking into account that (see (50), (99))

$$
\partial_{i} \ln u^{k}=\frac{\partial_{i} v^{k}}{v^{i}-v^{k}}, \quad i \neq k
$$

we obtain the expression

$$
\partial_{i} v^{i}=\sum_{m \neq i} \epsilon_{i m}\left(v^{m}-v^{i}\right) \partial_{i} u^{m}+\sum_{m \neq i} \epsilon_{i m} u^{m}\left(\partial_{i} v^{m}-\partial_{i} v^{i}\right),
$$

which reduces, on using (52), to the form

$$
\partial_{i} v^{i}\left(1+\sum_{m \neq i} \epsilon_{i m} u^{m}\right)=0 .
$$

Equation (108) can only be satisfied if $\partial_{i} v^{i}=0$ for all $i$ (otherwise the field variables $u^{m}$ in the algebraic system (38) would cease to be independent). Thus system (36), (38) is indeed linearly degenerate.

Now, differentiation of algebraic system (38) with respect to the Riemann invariant $r^{k}$ yields

$\partial_{k} v^{i}=\sum_{m \neq i, k} \epsilon_{i m} u^{m}\left(\partial_{k} v^{m}-\partial_{k} v^{i}\right)+\sum_{m \neq i, k} \epsilon_{i m}\left(v^{m}-v^{i}\right) \partial_{k} u^{m}+\epsilon_{i k} u^{k}\left(\partial_{k} v^{k}-\partial_{k} v^{i}\right)+\epsilon_{i k}\left(v^{k}-v^{i}\right) \partial_{k} u^{k}$, which reduces, with an account of (52) and the linear degeneracy property, to

$$
\left(v^{k}-v^{i}\right)\left[\left(1+\sum_{m \neq i} \epsilon_{i m} u^{m}\right) \partial_{k} \ln u^{i}-\sum_{m \neq i} \epsilon_{i m} \partial_{k} u^{m}\right]=0 .
$$

Since all characteristic velocities $v^{k}$ are distinct, the expression in square brackets must vanish for any pair of indices $i$ and $k$, i.e. we have

$$
\partial_{k} \ln u^{i}=\frac{\sum_{m \neq i} \epsilon_{i m} \partial_{k} u^{m}}{1+\sum_{m \neq i} \epsilon_{i m} u^{m}}, \quad k \neq i .
$$

Integration of (109) yields

$$
\sum_{m \neq i} \epsilon_{i m} u^{m}+R_{i}\left(r^{i}\right) u^{i}=-1
$$


where $R_{i}\left(r^{i}\right), i=1, \ldots, N$ are arbitrary functions.

We now differentiate (110) with respect to the Riemann invariants $r^{i}$ and $r^{k}$, which gives, on using (99) and (62),

$$
\sum_{m \neq i} \epsilon_{i m} \beta_{i m}+R_{i}^{\prime}\left(r^{i}\right)+R_{i}\left(r^{i}\right) \partial_{i} \ln \bar{H}_{i}=0
$$

and

$$
\sum_{m \neq i, k} \epsilon_{i m} \beta_{k m}+R_{i}\left(r^{i}\right) \beta_{k i}+\epsilon_{i k} \partial_{k} \ln \bar{H}_{k}=0
$$

respectively. Substitution of (106) into (101) gives

$$
\bar{H}_{i}=\sum_{m \neq i} \beta_{i m}+\partial_{i} \ln \bar{H}_{i}, \quad \tilde{H}_{i}=\xi_{i} \bar{H}_{i}+\sum_{m \neq i}\left(\xi_{m}-\xi_{i}\right) \beta_{i m}
$$

By expressing $\partial_{i} \ln \bar{H}_{i}$ from the above first equation, (111) and (112) reduce to the form

$$
\begin{aligned}
& R_{i}\left(r^{i}\right) \bar{H}_{i}=R_{i}\left(r^{i}\right) \sum_{m \neq i} \beta_{i m}-\sum_{m \neq i} \epsilon_{i m} \beta_{i m}-R_{i}^{\prime}\left(r^{i}\right), \\
& \epsilon_{i m} \bar{H}_{m}=\epsilon_{i m} \sum_{n \neq m} \beta_{n m}-\sum_{n \neq i, m} \epsilon_{i n} \beta_{n m}-R_{i}\left(r^{i}\right) \beta_{i m} .
\end{aligned}
$$

Substitution of the expressions $R_{i}\left(r^{i}\right) \bar{H}_{i}$ and $\epsilon_{i m} \bar{H}_{m}$ into (110) yields to a set of constraints $R_{i}^{\prime}\left(r^{i}\right)=1$, i.e. $R_{i}\left(r^{i}\right)=r^{i}+\alpha_{i}$, where $\alpha_{i}$ are arbitrary constants. Since any function of the Riemann invariant is a Riemann invariant as well one can put without loss of generality that $R_{i}\left(r^{i}\right)=r^{i}$. Then (110) reduces to (107). The Theorem is proved.

Taking into account $R_{i}\left(r^{i}\right)=r^{i}$ and eliminating $\bar{H}_{i}$ from (114) we arrive at the linear algebraic system

$$
\sum_{m \neq i, k}\left(r^{i} \epsilon_{k m}-\epsilon_{i k} \epsilon_{i m}\right) \beta_{i m}+\left(r^{i} r^{k}-\epsilon_{i k}^{2}\right) \beta_{i k}=\epsilon_{i k}, \quad i \neq k
$$

for the rotation coefficients $\beta_{i k}$, while (114) reduces (cf. the first formula in (113)) to

$$
\bar{H}_{i}=\sum_{m \neq i}\left(1-\frac{\epsilon_{i m}}{r^{i}}\right) \beta_{i m}-\frac{1}{r^{i}} .
$$

Let us introduce a matrix $\boldsymbol{\epsilon}$ such that its off-diagonal coefficients are the aforementioned symmetric constants $\epsilon_{i k}$, while the diagonal coefficients $\epsilon_{i i}=r^{i}$. 
Theorem 7.2: The rotation coefficients $\beta_{i k}$ satisfying linear algebraic system (115) are the off-diagonal components of the matrix inverse to the matrix - $\boldsymbol{\epsilon}$, i.e.

$$
\sum_{m=1}^{N} \epsilon_{i m} \beta_{k m}=-\delta_{i k} .
$$

Proof: We introduce the functions $\beta_{i i}(\mathbf{r})$ so that expression (63) could be extended to the full set of indices, i.e. we will have

$$
\partial_{i} \beta_{j k}=\beta_{j i} \beta_{i k} \quad \forall i, j, k
$$

It is easy to check that (118) is valid for any curvilinear coordinate system associated with semi-Hamiltonian Egorov linearly degenerate hydrodynamic type system (see (49) and (101)), i.e. if and only if the rotation coefficients $\beta_{i k}$ are symmetric (see (102)) and determined by (67), where the functions

$$
\beta_{i i}(\mathbf{r}) \equiv \partial_{i} \ln \bar{H}_{i}
$$

Indeed, the above set of equations (118) for two distinct indices (just two choices) reduces to the form

$$
\partial_{k} \beta_{j k}=\beta_{j k} \beta_{k k}, \quad \partial_{i} \beta_{k k}=\beta_{i k}^{2}
$$

The first part of these equations is nothing else but (67) while the second part is just the well-known property of any curvilinear coordinate net (see [10]): the scalar potential $V$ is determined by its second derivatives, i.e.

$$
\partial_{i k}^{2} V=\beta_{i k} \beta_{k i}, \quad k \neq i
$$

Thus, in the Egorov (symmetric) case, the above property (121) simplifies to

$$
\partial_{i k}^{2} V=\beta_{i k}^{2}, \quad k \neq i
$$

Comparing this formula and the second formula in (120), one can conclude that $\beta_{k k}=\partial_{k} V$. If all indices in (120) coincide, the last nontrivial consequence given by

$$
\partial_{k} \frac{1}{\beta_{k k}}=-1
$$


allows one to integrate (step-by-step) nonlinear system in partial derivatives (118). Instead of this direct, but somewhat complicated procedure, we shall use a more sophisticated but technically much more simple approach to the derivation of a general solution of system (118). First, let us introduce the combinations

$$
A_{i k}=\sum_{m=1}^{N} \epsilon_{i m} \beta_{k m}
$$

(we recall that $\epsilon_{i i}=r^{i}$ ). Then (115) reads as follows

$$
r^{i} A_{i k}=\epsilon_{i k}\left(1+A_{i i}\right), \quad i \neq k .
$$

Differentiation of (124) with respect to the Riemann invariants $r^{i}, r^{k}, r^{j}$ leads to the system

$$
\partial_{i} A_{i k}=\beta_{i k}\left(1+A_{i i}\right), \quad \partial_{k} A_{i k}=\beta_{k k} A_{i k}, \quad \partial_{j} A_{i k}=\beta_{j k} A_{i j}, \quad i \neq k .
$$

Compatibility conditions imply just one extra equation

$$
\partial_{k} A_{i i}=\beta_{i k} A_{i k}, \quad i \neq k .
$$

Now we differentiate (125) with respect to the Riemann invariant $r^{j}$ to obtain

$$
\left(r^{i} \beta_{j k}-\epsilon_{i k} \beta_{i j}\right) A_{i j}=0, \quad i \neq j \neq k
$$

Since expressions $r^{i} \beta_{j k}-\epsilon_{i k} \beta_{i j}$ cannot vanish identically, we have the only possible choice: $A_{i k}=0$ for each pair of distinct indices, and $A_{i i}=-1$ (see (125)). Thus, we conclude that (124) reduces to the form (117) (let us emphasize one more time that $\epsilon_{i i} \equiv r^{i}$, while all the other $\epsilon_{j k}=\epsilon_{k j}$ are constants). The matrix $\epsilon$ contains $N(N-1) / 2$ arbitrary constants $\epsilon_{i k}$, then all components of the matrix $\boldsymbol{\beta}$ are parameterized by these $N(N-1) / 2$ arbitrary constants. On the other hand, (118) is an overdetermined system, where all first derivatives of $\beta_{i k}$ are expressed via $\beta_{j n}$ only. Thus, a general solution of system in partial derivatives (118) must depend on $N(N+1) / 2$ arbitrary constants, because this system is written for $N(N+1) / 2$ functions $\beta_{i k}$ (these are $N(N-1) / 2$ symmetric off-diagonal elements, i.e. rotation coefficients $\beta_{i k}$; and $N$ diagonal components $\beta_{k k}$ ). It means, that the inverse matrix $\boldsymbol{\epsilon}$ contains extra $N$ arbitrary constants $\alpha_{i}$ which are nothing but the shifts of the Riemann invariants $r^{i}$ located 
on the diagonal (see the end of the proof of Theorem 7.1). Then these $N$ shift constants can be removed without loss of generality. The Theorem is proved.

In particular, for $N=3$ we have from (117) the explicit expressions for $\beta_{i k}$ :

$$
\begin{aligned}
& \beta_{12}=\frac{r^{3} \epsilon_{12}-\epsilon_{13} \epsilon_{23}}{r^{1} r^{2} r^{3}-r^{1} \epsilon_{23}^{2}-r^{2} \epsilon_{13}^{2}-r^{3} \epsilon_{12}^{2}+2 \epsilon_{12} \epsilon_{13} \epsilon_{23}}, \\
& \beta_{13}=\frac{r^{2} \epsilon_{13}-\epsilon_{12} \epsilon_{23}}{r^{1} r^{2} r^{3}-r^{1} \epsilon_{23}^{2}-r^{2} \epsilon_{13}^{2}-r^{3} \epsilon_{12}^{2}+2 \epsilon_{12} \epsilon_{13} \epsilon_{23}}, \\
& \beta_{23}=\frac{r^{1} \epsilon_{23}-\epsilon_{12} \epsilon_{13}}{r^{1} r^{2} r^{3}-r^{1} \epsilon_{23}^{2}-r^{2} \epsilon_{13}^{2}-r^{3} \epsilon_{12}^{2}+2 \epsilon_{12} \epsilon_{13} \epsilon_{23}} \\
& \beta_{11}=\frac{-r^{2} r^{3}+\epsilon_{23}^{2}}{r^{1} r^{2} r^{3}-r^{1} \epsilon_{23}^{2}-r^{2} \epsilon_{13}^{2}-r^{3} \epsilon_{12}^{2}+2 \epsilon_{12} \epsilon_{13} \epsilon_{23}}, \\
& \beta_{22}=\frac{-r^{1} r^{3}+\epsilon_{13}^{2}}{r^{1} r^{2} r^{3}-r^{1} \epsilon_{23}^{2}-r^{2} \epsilon_{13}^{2}-r^{3} \epsilon_{12}^{2}+2 \epsilon_{12} \epsilon_{13} \epsilon_{23}}, \\
& \beta_{33}=\frac{-r^{1} r^{2}+\epsilon_{12}^{2}}{r^{1} r^{2} r^{3}-r^{1} \epsilon_{23}^{2}-r^{2} \epsilon_{13}^{2}-r^{3} \epsilon_{12}^{2}+2 \epsilon_{12} \epsilon_{13} \epsilon_{23}} .
\end{aligned}
$$

Remark. Note that equation (117), despite of having a simpler form than original equation (115), is more general as it defines all (not only off-diagonal) components $\beta_{i k}$ in terms of Riemann invariants. Thus, the rotation coefficients $\beta_{i k}, i \neq k$ satisfy both systems (115) and (118) and are completely defined in terms of the matrix $\boldsymbol{\epsilon}$.

As a by-product of the proof of Theorem 7.2 we obtain the following important

Corollary 7.1 Since system (118) describes rotation coefficients $\beta_{i k}$ associated with hydrodynamic type systems possessing simultaneously Egorov and linear degeneracy properties, we conclude that our reduction (36), (38) of the kinetic equation (1) is the only (up to unessential transformations) hydrodynamic type system possessing both these properties.

Now, using (66), (99), (113), (116) and (119) we formulate the main result of this Section:

Algebraic relations (38) can be resolved in a parametric form in terms of the Riemann invariants:

$$
u^{i}=\sum_{m=1}^{N} \beta_{i m}, \quad v^{i}=\frac{1}{u^{i}} \sum_{m=1}^{N} \xi_{m} \beta_{i m},
$$

where the symmetric coefficients $\beta_{i k}$ are elements of the matrix $-\boldsymbol{\epsilon}^{-1}$ (see (117)). As a matter of fact, the first formula in (127) represents the inversion of formula (107). As one can see, this inversion is rather nontrivial. 
In particular, for $N=3$ we have from (127) the explicit expressions for conservation law densities $u^{i}$ and characteristic velocities $v^{k}$

$$
\begin{gathered}
u^{1}=\frac{-r^{2} r^{3}+r^{2} \epsilon_{13}+r^{3} \epsilon_{12}-\epsilon_{12} \epsilon_{23}-\epsilon_{13} \epsilon_{23}+\epsilon_{23}^{2}}{r^{1} r^{2} r^{3}-r^{1} \epsilon_{23}^{2}-r^{2} \epsilon_{13}^{2}-r^{3} \epsilon_{12}^{2}+2 \epsilon_{12} \epsilon_{13} \epsilon_{23}}, \\
u^{2}=\frac{-r^{1} r^{3}+r^{1} \epsilon_{23}+r^{3} \epsilon_{12}-\epsilon_{12} \epsilon_{13}-\epsilon_{13} \epsilon_{23}+\epsilon_{13}^{2}}{r^{1} r^{2} r^{3}-r^{1} \epsilon_{23}^{2}-r^{2} \epsilon_{13}^{2}-r^{3} \epsilon_{12}^{2}+2 \epsilon_{12} \epsilon_{13} \epsilon_{23}}, \\
u^{3}=\frac{-r^{1} r^{2}+r^{1} \epsilon_{23}+r^{2} \epsilon_{13}-\epsilon_{12} \epsilon_{13}-\epsilon_{12} \epsilon_{23}+\epsilon_{12}^{2}}{r^{1} r^{2} r^{3}-r^{1} \epsilon_{23}^{2}-r^{2} \epsilon_{13}^{2}-r^{3} \epsilon_{12}^{2}+2 \epsilon_{12} \epsilon_{13} \epsilon_{23}}, \\
v^{1}=\frac{\xi_{1}\left(\epsilon_{23}^{2}-r^{2} r^{3}\right)+\xi_{2}\left(r^{3} \epsilon_{12}-\epsilon_{13} \epsilon_{23}\right)+\xi_{3}\left(r^{2} \epsilon_{13}-\epsilon_{12} \epsilon_{23}\right)}{\epsilon_{23}^{2}-r^{2} r^{3}+\epsilon_{13}\left(r^{2}-\epsilon_{23}\right)+\epsilon_{12}\left(r^{3}-\epsilon_{23}\right)}, \\
v^{2}=\frac{\xi_{2}\left(\epsilon_{13}^{2}-r^{1} r^{3}\right)+\xi_{3}\left(r^{1} \epsilon_{23}-\epsilon_{12} \epsilon_{13}\right)+\xi_{1}\left(r^{3} \epsilon_{12}-\epsilon_{13} \epsilon_{23}\right)}{\epsilon_{13}^{2}-r^{1} r^{3}+\epsilon_{13}\left(r^{3}-\epsilon_{13}\right)+\epsilon_{23}\left(r^{1}-\epsilon_{13}\right)}, \\
v^{3}=\frac{\xi_{3}\left(\epsilon_{12}^{2}-r^{1} r^{2}\right)+\xi_{2}\left(r^{1} \epsilon_{23}-\epsilon_{12} \epsilon_{13}\right)+\xi_{1}\left(r^{2} \epsilon_{13}-\epsilon_{12} \epsilon_{23}\right)}{\epsilon_{12}^{2}-r^{1} r^{2}+\epsilon_{13}\left(r^{2}-\epsilon_{12}\right)+\epsilon_{23}\left(r^{1}-\epsilon_{12}\right)} .
\end{gathered}
$$

One can observe that formulae (129) do not coincide with representation (78), (76) obtained earlier for the same family of the characteristic velocities. The reason is that the two representations correspond to different choices of the Riemann invariants (we recall one more time that any function of a Riemann invariant is a Riemann invariant as well). The relationship between these two equivalent sets of the Riemann invariants can be obtained by equating the characteristic velocities (78) and (129) (or, alternatively, the densities (79) and (128)), which do not depend on a particular normalization of the Riemann invariants. It is more convenient, however, to get the sought relationship by a substitution (128) into (82), where we replace $r_{i}$ with $\tilde{r}_{i}$. As a result we get

$$
\begin{aligned}
\tilde{r}^{1} & =\frac{\left(\epsilon_{13}-\epsilon_{12}\right)\left(\epsilon_{23} r^{1}-\epsilon_{12} \epsilon_{13}\right)}{\left(\xi_{2}-\xi_{3}\right) r^{1}+\left(\xi_{3}-\xi_{1}\right) \epsilon_{12}+\left(\xi_{1}-\xi_{2}\right) \epsilon_{13}}, \\
\tilde{r}^{2} & =\frac{\left(\epsilon_{12}-\epsilon_{23}\right)\left(\epsilon_{13} r^{2}-\epsilon_{12} \epsilon_{23}\right)}{\left(\xi_{3}-\xi_{1}\right) r^{2}+\left(\xi_{1}-\xi_{2}\right) \epsilon_{23}+\left(\xi_{2}-\xi_{3}\right) \epsilon_{12}}, \\
\tilde{r}^{3} & =\frac{\left(\epsilon_{23}-\epsilon_{13}\right)\left(\epsilon_{12} r^{3}-\epsilon_{13} \epsilon_{23}\right)}{\left(\xi_{1}-\xi_{2}\right) r^{3}+\left(\xi_{2}-\xi_{3}\right) \epsilon_{13}+\left(\xi_{3}-\xi_{1}\right) \epsilon_{23}} .
\end{aligned}
$$

Here by $\tilde{r}^{1}, \tilde{r}^{2}, \tilde{r}^{3}$ we denote the 'old' Riemann invariants as in Section 5.

Note that the choice (130) of the Riemann invariants leads to the homogeneous expressions (78) for the characteristic velocities, which makes possible the construction of the 
similarity solutions (85). Such a possibility, however, is unique to the case $N=3$ as for $N>3$ the general rational substitution of the type (130) will not allow for the elimination of all inhomogeneous terms (unless one has a very special set of the coefficients $\epsilon_{i j}, \xi_{k}$ ). As a consequence, the family of the similarity solutions (83) exists only for the case $N=3$ which makes this case special.

\section{Commuting hydrodynamic flows}

\subsection{General explicit representation}

Commuting flows to semi-Hamiltonian linearly degenerate system (36), (38) are defined in terms of the Riemann invariants by equations (48), (47). We recall that, according to Proposition 4.1, only $N-2$ of the commuting flows are linearly degenerate (excluding the 'trivial' flows specified by linear combinations of the constant characteristic velocity $\mathbf{1}$ and the characteristic velocity $\mathbf{v}$ of the original flow (44)). The general solution of the generalised hodograph equations (47) specifying commuting flows was obtained by Ferapontov [11] in terms of the Stäckel matrix entries (see Theorem 4.2). Here we are interested in a more explicit representation of the commuting flows for the specific system (36), (38). For that, instead of integrating system (47), we take advantage of the fact that our linearly degenerate system (36), (38) is Egorov. In that case, the commuting flows can be found explicitly.

We first observe that any conservation law density $h$ for linearly degenerate hydrodynamic type system (36) can be represented in the form (see (53) or (57))

$$
h=\sum_{k=1}^{N} u^{k} P_{k}\left(r^{k}\right)
$$

with $N$ arbitrary functions $P_{k}\left(r^{k}\right)$ of a single variable. Then we make use of

Lemma 8.1 (Pavlov \& Tsarev 2003 [38]): All commuting flows (48), (47) in the Egorov case are specified by the expression (see (65), (101), (103))

$$
W^{i}=\frac{H_{i}}{\bar{H}_{i}}=\frac{\partial_{i} h}{\partial_{i} a} .
$$


Substituting (131), (106) into (132) and using the first formula from (113) we obtain an explicit representation for the characteristic velocities of the commuting flows (48),

$$
W^{i}=P_{i}\left(r^{i}\right)+\frac{1}{\bar{H}_{i}}\left(P_{i}^{\prime}\left(r^{i}\right)+\sum_{m \neq i}\left(P_{m}\left(r^{m}\right)-P_{i}\left(r^{i}\right)\right) \beta_{i m}\right) .
$$

We recall that $P_{k}\left(r^{k}\right), k=1, \ldots, N$ are arbitrary functions and the dependence of the rotation coefficients $\beta_{i m}$ on the Riemann invariants is found by inversion of the matrix $-\boldsymbol{\epsilon}$ (see $(117))$. If $P_{k}\left(r^{k}\right)=1$, (133) reduces to $W^{i}=1$; if $P_{k}\left(r^{k}\right)=\xi_{k}$, it reduces to the second formula in (127), i.e. to hydrodynamic reduction (36), (38) itself.

\subsection{Generalized hodograph method}

Taking into account the Combescure transformation (65) and formula (66) the generalized hodograph solution (46) can be represented in a symmetric form

$$
x \bar{H}_{i}+t \tilde{H}_{i}=H_{i}(\mathbf{r})
$$

Since (see (127), (66), (99))

$$
\bar{H}_{i}=\sum_{m=1}^{N} \beta_{i m}, \quad \tilde{H}_{i}=\sum_{m=1}^{N} \xi_{m} \beta_{i m},
$$

expression (134), with an account of (65), (133), assumes the form

$$
\sum_{m=1}^{N}\left(x+\xi_{m} t\right) \beta_{k m}=P_{k}^{\prime}\left(r^{k}\right)+\sum_{m=1}^{N} P_{m}\left(r^{m}\right) \beta_{k m} .
$$

Multiplying equation (135) through by the matrix $\boldsymbol{\epsilon}$ and performing summation, $\sum_{k=1}^{N} \epsilon_{i k}[\ldots]_{k}$, we obtain, upon using (117), a general solution of the $N$-component linearly degenerate hydrodynamic reduction in an implicit form (cf. (61))

$$
x+\xi_{i} t=P_{i}\left(r^{i}\right)-r^{i} P_{i}^{\prime}\left(r^{i}\right)-\sum_{m \neq i} \epsilon_{i m} P_{m}^{\prime}\left(r^{m}\right), \quad i=1,2, \ldots, N,
$$

where $P_{i}\left(r^{i}\right), i=1, \ldots, N$, are arbitrary functions.

Note that under the re-parametrization

$$
P_{k}^{\prime \prime}(\xi)=-\frac{\phi_{k}(\xi)}{f(\xi)}
$$


the generalized hodograph solution (136) becomes

$$
x+\xi_{i} t=\int^{r^{i}} \frac{\xi \phi_{i}(\xi)}{f(\xi)} d \xi+\sum_{m \neq i} \epsilon_{i m} \int^{r^{m}} \frac{\phi_{m}(\xi)}{f(\xi)} d \xi
$$

Now, comparison of (137) with the Ferapontov [11] solution (61) provides a direct way for the identification of the entries of the Stäckel matrix (55). Also, for this choice of the Stäckel matrix all constants $C_{l, m}$ (see Section 6) can be expressed in terms of the coefficients $\epsilon_{i j}$ and $\xi_{k}$ by (97), (98).

For the particular choice of $f(\xi)$ defined as

$$
f(\xi)=\sqrt{R_{K}(\xi)}
$$

where

$$
R_{K}(\xi)=\prod_{n=1}^{K}\left(\xi-E_{n}\right)
$$

and $E_{1}<E_{2}<\cdots<E_{K}$ are real constants $(K=2 N+1$ if $N$ is odd and $K=2 N+2$ if $N$ is even); and $\phi_{k}(\xi)$ being arbitrary polynomials in $\xi$ of degrees less than $N$, system (136) describes quasiperiodic solutions of the form

$$
x+\xi_{i} t=\int^{r^{i}} \frac{\xi \phi_{i}(\xi) d \xi}{\sqrt{R_{K}(\xi)}}+\sum_{m \neq i} \epsilon_{i m} \int^{r^{m}} \frac{\phi_{m}(\xi) d \xi}{\sqrt{R_{K}(\xi)}}, \quad i=1,2, \ldots, N
$$

The proof of quasiperiodicity of solution (139) is analogous to that for solution (86), (87), (88) obtained for $N=3$.

\subsection{Linearly degenerate commuting flows}

To extract the family of linearly degenerate commuting flows from general representation (133) we formulate the following

Lemma 8.2: For the linearly degenerate commuting flows each function $P_{i}\left(r^{i}\right)$ in (133) is linear with respect to the corresponding Riemann invariant $r^{i}$.

Proof: The condition $\partial_{i} W^{i}=0$ of linear degeneracy of the commuting flow implies, on using (67) and (113), that $P_{i}^{\prime \prime}\left(r^{i}\right)=0$. 
We now consider the representation for the family of linearly degenerate commuting flows suggested by the form of the kinetic equations (33), (34) for the KdV hierarchy. Importantly, the whole KdV kinetic hierarchy (33), (34) is characterised by a single integral kernel, $G(\eta, \mu)=\ln |(\eta-\mu) /(\eta+\mu)|$ (which is consistent with the fact that all equations of the original finite-gap Whitham hierarchy are associated with the same Riemann surface). This suggests that there could exist a family of commuting flows to general nonlocal kinetic equation (1) having the form

$$
\begin{aligned}
f_{\tau} & =(\tilde{s} f)_{x} \\
\tilde{s}(\eta) & =\tilde{S}(\eta)+\frac{1}{\eta} \int_{0}^{\infty} G(\eta, \mu) f(\mu)[\tilde{s}(\mu)-\tilde{s}(\eta)] d \mu,
\end{aligned}
$$

where $\tilde{S}(\eta)$ is an arbitrary function. Although verification of commutativity of the kinetic equations (1) and (140) is beyond the scope of the present paper, it is clear that, if these equation do commute, this must be manifested on the level of hydrodynamic reductions as well. Having this in mind, we consider the $N$-component hydrodynamic reductions to (140) obtained by the familiar delta-functional ansatz (35) and try to see if they commute with the original reductions (36)-(39).

First we notice that equation (140) is, essentially, the same kinetic equation (1) but with a different time variable and different "free soliton speed" function $S(\eta)$. Now, since we have proved integrability of the linearly degenerate hydrodynamic reductions (36)-(39) in a general form, we automatically have that analogous $N$-component hydrodynamic reductions of (140) must also be integrable linearly degenerate systems. It should be noted that, since the function $\tilde{S}(\eta)$ is arbitrary, the set $\left\{\tilde{\xi}_{1}, \ldots, \tilde{\xi}_{N}\right\}$ of its values $\tilde{\xi}_{j}=\tilde{S}\left(\eta_{j}\right)$ can be viewed as a set of $N$ arbitrary numbers, and the corresponding 'cold-gas' hydrodynamic reduction becomes (cf. (36)-(39))

$$
u_{\tau}^{i}=\left(u^{i} \tilde{v}^{i}\right)_{x}, \quad i=1, \ldots, N
$$

where the velocities $\tilde{v}^{i}=-\tilde{s}^{i}$ and the conservation law densities $u^{i}$ satisfy algebraic relations

$$
\tilde{v}^{i}=\tilde{\xi}_{i}+\sum_{k \neq i}^{N} \epsilon_{i k} u^{k}\left(\tilde{v}^{k}-\tilde{v}^{i}\right), \quad \epsilon_{i k}=\epsilon_{k i}
$$


and $\epsilon_{i k}$ are the same as in (39).

According to Theorem 3.1, equations (141), (142) can be represented in the Riemann form

$$
r_{\tau}^{i}=\tilde{v}^{i}(\mathbf{r}) r_{x}^{i}, \quad i=1,2, \ldots, N
$$

where the dependence $\tilde{v}^{i}(\mathbf{r})$ of the characteristic velocities on the Riemann invariants is determined by the same formulae (127) with the only difference that, one now replaces $\xi_{j}$ with $\tilde{\xi}_{j}$, i.e. we have

$$
\tilde{v}^{i}=\frac{1}{u^{i}} \sum_{m=1}^{N} \tilde{\xi}_{m} \beta_{i m} .
$$

Indeed, representation (144) is a straightforward consequence of (127) since the rotation co-

efficients $\beta_{i j}$ and Lamé coefficients $\bar{H}_{k}$ do not depend on the parameters $\xi_{m}$ (see (115), (116), the first formula in (127), and normalisation (99)). It not difficult to see that commutativity relationships (see (47))

$$
\frac{\partial_{i} \tilde{v}^{j}}{\tilde{v}^{i}-\tilde{v}^{j}}=\frac{\partial_{i} v^{j}}{v^{i}-v^{j}}, \quad i, j=1,2, \ldots, N, \quad i \neq j
$$

are satisfied identically. Thus, we have proved the following

Lemma 8.3: Linearly degenerate semi-Hamiltonian flows (141), (142) and (36), (38) commute for any $N$.

In conclusion we note that, although we have proved integrability of the 'cold-gas' hydrodynamic reductions (36), (38) for an arbitrary choice of the functions $S(\eta)$ and $G(\eta, \mu)$ in the original kinetic equation (1), one can expect that integrability of the full equation (1) would require some additional restrictions imposed on the integral kernel $G(\eta, \mu)$ (other than just symmetry).

\section{Outlook and Perspectives}

Kinetic equation (1) first arose as a continuum (thermodynamic) limit of a semi-Hamiltonian hydrodynamic type system (the KdV-Whitham system). This equation seems to belong to an entirely new class of integrable systems, which we at present are unable to equip with the 
standard attributes such as a Lax pair, commuting flows, Hamiltonian structures etc. This paper makes the first step towards the understanding of the integrable structure of equation (1) by studying in detail the simplest class of its hydrodynamic reductions and identifying them as Egorov, semi-Hamiltonian linearly degenerate hydrodynamic type systems. The availability of an infinite set of the aforementioned hydrodynamic reductions is a strong evidence that the full equation (1) could constitute an integrable system in the sense yet to be explored. While the studied 'cold-gas' reductions turn out to be integrable for an arbitrary symmetric 'interaction kernel' $G(\eta, \mu)$, integrability of the full equation (1) will clearly require some additional restrictions to be imposed on this kernel. Recent results $[12,13],[18,19]$ on the integrability of $2+1$ hydrodynamic type systems and hydrodynamic chains, which are close 'relatives' of kinetic equations, suggest that these restrictions should be determined by the condition of the existence, for an arbitrary $N$, of $N$-component hydrodynamic reductions parameterized by $N$ arbitrary functions of a single variable. The most natural way to attack this problem is to study the associated hydrodynamic chain, i.e. an infinite set of the moment equations (see e.g. [20]) for kinetic equation (1). However, due to the structure of the nonlocal term in (1) the construction of this chain is far from being a straightforward task.

The study of the moment equations for (1) is also important in the original context of the description of macroscopic dynamics of soliton gases [46], [7]. Indeed, the kinetic description of a soliton gas reflects the particle-like nature of solitons. At the same time, one should remember that solitons represent localized waves so the kinetic description of a soliton gas should be complemented by the expressions for the averaged characteristics of the underlying 'microscopic' oscillatory wave field in terms of the distribution function $f(\eta, x, t)$. Say, for the $\mathrm{KdV}$ equation (3) the expressions for the two first moments of the wave field have the form (see [6])

$$
\bar{\phi}(x, t)=4 \int_{0}^{\infty} \eta f(\eta, x, t) d \eta, \quad \overline{\phi^{2}}(x, t)=\frac{16}{3} \int_{0}^{\infty} \eta^{3} f(\eta, x, t) d \eta
$$

and are identical to those arising in the Lax-Levermore-Venakides theory [31], [43], [45] with the crucial difference that the dynamics of the distribution function $f(\eta, x, t)$ is now governed by kinetic equation (1), (2) rather than the $N$-phase averaged Whitham equations (10) so 
(146) are ensemble averages.

This paper was concerned mostly with the structure of the kinetic equation (1). At the same time, behaviour of its solutions and the associated evolution of the dynamical (moments, amplitudes etc.) and probabilistic (probability density, correlation function etc.) characteristics of the underlying rapidly oscillating wave field could be of considerable interest for applications. In this regard, we mention an interesting consideration following from our present study. In the original construction [6] described in Section 3 the kinetic equation for the $\mathrm{KdV}$ soliton gas was obtained as the thermodynamic limit of the $N$-phase averaged KdV-Whitham equations (10). These Whitham equations are genuinely nonlinear for any $N \in \mathbb{N}$ [32], i.e. for a reasonably general class of initial conditions the modulation dynamics specified on a Riemann surface of genus $N$ implies hydrodynamic breaking at some $t<\infty$ accompanied by the growth of the genus $N$ (see e.g. [5], [9], [22]). At the same time, the 'coldgas' hydrodynamic reductions of the kinetic equations studied here are linearly degenerate, i.e. no breaking is expected and the number of gas components does not change during the evolution (a simple example of such non-breaking evolution for a two-component soliton gas was considered in [7]). Of course, there is no contradiction between these two contrasting types of behaviour as the kinetic equation (28), (26) represents a singular limit as $N \rightarrow \infty$ of the $\mathrm{KdV}$-Whitham equations while their genuine nonlinearity property is established only for finite $N$. Construction of physical solutions to linearly degenerate multi-component hydrodynamic type system (4), (5) and study of the associated wave field dynamics of soliton gases in various integrable systems represents a separate interesting mathematical problem, which could find applications in the description of propagation and interaction of quasi-monochromatic soliton beams in dispersive dissipationless media.

Another challenging problem is derivation of the $2+1$ dimensional kinetic equation for the soliton gas in the framework of the Kadomtsev - Petviashvili (KP-2) equation. This problem would require computing the thermodynamic limit of the KP-Whitham equations associated with general algebraic (not necessarily hyperelliptic) Riemann surfaces [25], [26].

Finally, we would like to mention one more perspective arising from our study. To our best knowledge, nonlocal kinetic equation (1) is the first available example of a continuum 
limit of a semi-Hamiltonian hydrodynamic type system. The key point of its derivation is that it is not sufficient to simply tend the number of Riemann invariants to infinity but it is important to prescribe a special scaling controlling the distances between neighboring invariants (in the case of averaged finite-gap dynamics, the widths of spectral bands and gaps - see (19)). We believe that a similar approach could be applied to a large class of semi-Hamiltonian hydrodynamic type systems (not necessarily arising as the result of the Whitham averaging). Of course, the corresponding thermodynamic scaling (an analogue of distribution (19)) could be different.

\section{Acknowledgments}

We are grateful to V.E. Zakharov for his interest in this work and a number of enlightening comments. We thank Yu. Fedorov, E. Ferapontov, O. Morozov, A. Neishtadt, Z. Popowicz, S. Tsarev and A. Veselov for stimulating discussions. The work has been partially supported by EPSRC (UK)(grant EP/E040160/1) and London Mathematical Society (Scheme 4 Collaborative Visits Grant). Work of M.V.P. has been also supported by the Programme "Fundamental problems of nonlinear dynamics" of Presidium of RAS. M.V.P. and S.A.Z. also acknowledge partial financial support from the Russian-Taiwanese grant 95WFE0300007 (RFBR grant 06-01-89507-HHC) .

\section{References}

[1] Arik, M., Neyzi, F., Nutku, Ya. and Olver, P., Multi-Hamiltonian structure of the Born-Infeld equation, J. Math. Phys. 30 (1989) 1338-1344.

[2] Belokolos, E.D., Kinetic equations and integrable Hamiltonian systems, Ukr. Math. Journ. 57 (2005) $869-882$.

[3] Benney, D.J., Some properties of long nonlinear waves, Stud. Appl. Math. 52 (1973) $45-50$. 
[4] Born, M. and Infeld, L., Foundations of a new field theory, Proc. Roy. Soc. A 144 (1934) 425-451.

[5] Dubrovin, B.A. and Novikov, S.P., Hydrodynamics of weakly deformed soliton lattices. Differential geometry and Hamiltonian theory, Russian Math. Surveys 44 (1989) 35-124.

[6] El, G.A., The thermodynamic limit of the Whitham equations, Phys. Lett. A 311 (2003) $374-383$.

[7] El, G.A. and Kamchatnov, A.M., Kinetic equation for a dense soliton gas, Phys. Rev. Lett. 95 (2005) Art. No. 204101.

[8] El, G.A., Krylov, A.L., Molchanov, S.A. and Venakides, S., Soliton turbulence as the thermodynamic limit of stochastic soliton lattices, Advances in Nonlinear Mathematics and Science, Physica D 152-153 (2001) 653-664.

[9] El, G.A., Krylov, A.L. and Venakides, S., Unified approach to KdV modulations, Comm. Pure Appl. Math. 54 (2001) 1243-1270.

[10] Darboux G., Leçons sur les systèmes orthogonaux et les coordonnées curvilignes, Paris (1910).

[11] Ferapontov, E.V., Integration of weakly-nolinear hydrodynamic systems in Riemann invariants, Phys. Lett. A 158 (1991) 112-118.

[12] Ferapontov, E.V. and Khusnutdinova, K.R., On integrability of $(2+1)$-dimensional quasilinear systems, Comm. Math. Phys. 248 (2004) 187-206.

[13] Ferapontov, E.V. and Khusnutdinova, K.R., The characterization of 2-component $(2+1)$-dimensional integrable systems of hydrodynamic type, J. Phys. A: Math. Gen. 37 (2004) 2949 - 2963.

[14] Ferapontov, E.V. and Marshall, D.G., Differential-geometric approach to the integrability of hydrodynamic chains: the Haantjes tensor, Matematische Annalen 339 (2007) $61-99$. 
[15] Flaschka, H., Forest, G., McLaughlin, D.W., Multiphase averaging and the inverse spectral solutions of the Korteweg - de Vries equation, Comm. Pure Appl. Math. 33 (1980) 739-784.

[16] Gantmaher, F.R., Applications of the theory of matrices, Interscience, New York (1959).

[17] Gibbons, J., Collisionless Boltzmann equations and integrable moment equations, Physica D 3 (1981) 503-511.

[18] Gibbons, J. and Tsarev, S.P., Reductions of Benney's equations, Phys. Lett. A, 211 (1996) 19-24.

[19] Gibbons, J. and Tsarev, S.P., Conformal maps and reductions of the Benney equations, Phys. Lett. A, 258 (1999) 263-270.

[20] Gibbons, J. and Raimondo, A., Differential geometry of hydrodynamic Vlasov equations, Journ. Geom. Phys. 57 (2007) 1815-1828.

[21] Gibbons, J. and Kodama, Yu., Solving dispersionless Lax equations. In N. Ercolani et al., editor, Singular limits of dispersive waves, v. 320 of NATO ASI Series B, page 61. Plenum, New York (1994).

[22] Grava, T. and Tian,F.-R. The generation, propagation, and extinction of multiphases in the KdV zero-dispersion limit, Comm. Pure Appl. Math. 55 (2002) 1569-1639 .

[23] Gurevich, A.V., Mazur, N.G., and Zybin, K.P., Statistical limit in a completely integrable system with deterministic initial conditions, Journ. Exp. Theor. Phys. 90 (2000) 797 - 817.

[24] Johnson, R. and Moser, J., The rotation number for almost periodic potentials, Comm. Math. Phys. 84 (1982) 403-438.

[25] Krichever, I.M., The averaging method for two-dimensional "integrable" equations, Funct. Anal. Appl. 22 (1988) 200-213. 
[26] Krichever, I.M., Spectral theory of two-dimensional periodic operators and its applications, Russian Math. Surveys 44 (1989) 145-225.

[27] Kodama, Yu., A method for solving the dispersionless KP equation and its exact solutions, Phys. Lett. A 129 (1988) 223-226.

[28] Kodama, Yu., A solution method for the dispersionless KP equation, Prog. Theor. Phys. Supplement. 94 (1988) 184.

[29] Kotani, S., KdV flow on generalized reflectionless potentials, Journ. of Math. Phys. Anal. Geom. 4 (2008) 490 - 528.

[30] Lax, P.D., The zero dispersion limit, a deterministic analog of turbulence, Comm. Pure Appl. Math. 44 (1991) 1047-1056.

[31] Lax, P.D. and Levermore, C.D., The small dispersion limit of the Korteweg - de Vries equation I, II, III, Comm. Pure Appl. Math. 36 (1983) 253-290, 571-593, 809-829.

[32] Levermore, C.D. The hyperbolic nature of the zero dispersion KdV limit. Comm. Partial Differential Equations 13 (1988) 495-514.

[33] Novikov, S.P., Manakov, S.V., Pitaevskii, L.P. and Zakharov, V.E., The Theory of Solitons: The Inverse Scattering Method, Consultants, New York (1984).

[34] Odesskii, A.V., Pavlov, M.V. and Sokolov, V.V. Classification of integrable Vlasov-like equations, Theor. Math. Phys. 154 (2008) 248-259.

[35] Pavlov, M.V., Hamiltonian formalism of weakly nonlinear systems in hydrodynamics, Theor. Math. Phys. 73 (1987) 1242-1245.

[36] Pavlov, M.V., Algebro-geometric approach in the theory of integrable hydrodynamic type systems, Comm. Math. Phys. 272 (2007) 469-505.

[37] Pavlov, M.V., Svinolupov, R.A. and Sharipov, S.I., Invariant integrability criterion for equations of hydrodynamic type, Func. Anal. Appl. 30 (1996) 15-22. 
[38] Pavlov, M.V. and Tsarev, S.P., Three-Hamiltonian structures of the Egorov hydrodynamic type systems, Funct. Anal. Appl., 37, No. 1 (2003) 32-45.

[39] Tsarev, S.P., On Poisson brackets and one-dimensional Hamiltonian systems of hydrodynamic type, Soviet Math. Dokl. 31 (1985) 488-491.

[40] Tsarev, S.P., The geometry of Hamiltonian systems of hydrodynamic type. The generalized hodograph method, Math. USSR Izvestiya 37 (1991) 397-419.

[41] Weinstein, M. I., and Keller, J. B., Asymptotic behavior of stability regions for Hills equation, SIAM Journal Appl. Math. 47 (1987) 941-958.

[42] Whitham, G.B., Linear and Nonlinear Waves, Wiley, New York (1974).

[43] Venakides, S., The zero dispersion limit of the Korteweg-de Vries equation with periodic initial data, AMS Transactions, vol. 301 (1987), pp. 189-226.

[44] Venakides, S., The continuum limit of theta functions, Comm.Pure Appl.Math. 42 (1989) 711.

[45] Venakides, S., The Korteweg-de Vries equation with small dispersion: Higher order Lax-Levermore theory, Comm.Pure Appl.Math. 43 (1990), pp. 335-361.

[46] Zakharov, V.E., Kinetic equation for solitons, Sov. Phys. JETP 33 (1971) 538-541.

[47] Zakharov, V.E., On the Benney equations, Physica D 3 (1981) 193-202.

[48] Zakharov, V.E., Dispersionless limit of integrable systems in $2+1$ dimensions, in Singular Limits of Dispersive Waves, Ed. N.M. Ercolani et al., Plenum Press, NY (1994) 165-174.

[49] Zakharov, V.E., Turbulence in integrable systems, Stud. Appl. Math. 122 (2009) 219 -234 . 